\title{
Non-Smooth Algorithms for Minimizing the Largest Eigenvalue with Applications to Inner Numerical Radius
}

\author{
FATIH Kangal $\dagger$ AND EMre MEngi $\ddagger$ \\ Department of Mathematics, Koç University \\ Rumelifeneri Yolu, 34450 Sarlyer-Istanbul, Turkey
}

[Received on 1 August 2019]

\begin{abstract}
Non-smoothness at optimal points is a common phenomenon in many eigenvalue optimization problems. We consider two recent algorithms to minimize the largest eigenvalue of a Hermitian matrix dependent on one parameter, both proven to be globally convergent unaffected by non-smoothness. One of these models the eigenvalue function with a piece-wise quadratic function, and is effective in dealing with non-convex problems. The other projects the Hermitian matrix into subspaces formed of eigenvectors, and is effective in dealing with large-scale problems. We generalize the latter slightly to cope with nonsmoothness. For both algorithms, we analyze the rate-of-convergence in the non-smooth setting, when the largest eigenvalue is multiple at the minimizer and zero is strictly in the interior of the generalized Clarke derivative, and prove that both algorithms converge rapidly. The algorithms are applied to, and the deduced results are illustrated on the computation of the inner numerical radius, the modulus of the point on the boundary of the field of values closest to the origin, which carries significance for instance for the numerical solution of a symmetric definite generalized eigenvalue problem and the iterative solution of a saddle point linear system.
\end{abstract}

Keywords: eigenvalue optimization; non-smooth optimization; global optimization; rate-of-convergence; subspace projections; field of values; definite matrix pairs; inner numerical radius

\section{Introduction}

A pair of Hermitian matrices $A, B \in \mathbb{C}^{n \times n}$ is said to be definite if

$$
\begin{aligned}
\gamma(A, B) & :=\min _{z \in \mathbb{C}^{n},\|z\|_{2}=1} \sqrt{\left(z^{*} A z\right)^{2}+\left(z^{*} B z\right)^{2}} \\
& =\min _{z \in \mathbb{C}^{n},\|z\|_{2}=1}\left|z^{*}(A+\mathrm{i} B) z\right|=\min \{|w| \mid w \in F(A+\mathrm{i} B)\}>0,
\end{aligned}
$$

where $F(C)$ denotes the field of values of $C \in \mathbb{C}^{n \times n}$, the subset of $\mathbb{C}$ defined by

$$
F(C):=\left\{z^{*} C z \in \mathbb{C} \mid z \in \mathbb{C}^{n},\|z\|_{2}=1\right\} .
$$

The definiteness of $(A, B)$ carries significance for the numerical solution of the generalized eigenvalue problem $A x=\lambda B x$ (Cheng \& Higham (1999); Davies et al. (2001)). If $(A, B)$ is known to be definite, the generalized eigenvalue problem can be transformed into another related problem $\widetilde{A} x=\lambda \widetilde{B} x$ with $\lambda_{\min }(\widetilde{B})=\gamma(A, B)$; indeed the eigenvalues of the original problem and the transformed problem are

${ }^{\dagger}$ Email: fkangal@ku.edu.tr

${ }^{\ddagger}$ Corresponding author. Email: emengi@ku.edu.tr 
related by a rotation. The transformed problem can be solved by calculating a Cholesky factorization $\widetilde{B}=R^{*} R$, and computing the eigenvalues of the Hermitian matrix $R^{-*} \widetilde{A} R^{-1}$. This is a plausible procedure with a small backward error provided $\gamma(A, B)$ is not small. Two other application areas concern Hermitian quadratic eigenvalue problems, in particular checking the hyperbolicity of such problems (Higham et al. (2002)), and saddle point linear systems with symmetric indefinite coefficient matrices, in particular setting up a conjugate gradient iteration for such systems (Liesen \& Parlett (2008)).

Motivated by such applications, Cheng and Higham have focused on procedures for efficient determination of whether a given pair $(A, B)$ of Hermitian matrices is definite or not (see Cheng \& Higham (1999)). If the Hermitian pair is not definite, in the same paper, the authors have also considered the computation of a pair $\left(A+\Delta A_{*}, B+\Delta B_{*}\right)$ where $\left(\Delta A_{*}, \Delta B_{*}\right)$ solves the following minimization problem for a prescribed positive real number $\delta$ :

$$
d_{\delta}(A, B):=\min \left\{\left\|\left[\begin{array}{ll}
\Delta A & \Delta B
\end{array}\right]\right\|_{2} \mid \gamma(A+\Delta A, B+\Delta B) \geqslant \delta\right\} .
$$

It is shown in Cheng \& Higham (1999) that whether the pair $(A, B)$ is definite or not, and, if it is not definite, the optimal $\left(\Delta A_{*}, \Delta B_{*}\right)$ can be determined by the global minimizer of

$$
\min _{\theta \in[0,2 \pi]} \lambda_{\max }(A \cos \theta+B \sin \theta)
$$

where and elsewhere $\lambda_{\max }(\cdot)$ represents the largest eigenvalue of its matrix argument. The eigenvalue optimization problem in (1.3) is non-convex, indeed it is argued in Cheng \& Higham (1999) that the major challenge for the estimation of $d_{\delta}(A, B)$ and the optimizer $\left(\Delta A_{*}, \Delta B_{*}\right)$ is the global solution of this problem. Geometrically, $\zeta(A, B):=\left|\min _{\theta \in[0,2 \pi]} \lambda_{\max }(A \cos \theta+B \sin \theta)\right|$ corresponds to the inner numerical radius of $A+\mathrm{i} B$, the modulus of the point on the boundary of $F(A+\mathrm{i} B)$ closest to the origin.

The optimization problem in (1.3) is only a special instance of a family of eigenvalue optimization problems

$$
\min _{\omega \in \Omega} \lambda_{\max }(\mathscr{A}(\omega)), \quad \mathscr{A}(\omega):=\sum_{j=1}^{\kappa} f_{j}(\omega) A_{j}
$$

where $\Omega$ is a closed interval in $\mathbb{R}$, the matrices $A_{1}, \ldots, A_{\kappa} \in \mathbb{C}^{n \times n}$ are Hermitian, and the functions $f_{1}, \ldots, f_{\kappa}: \underline{\Omega} \rightarrow \mathbb{R}$ are real analytic on their domain $\underline{\Omega}$, which is an open interval in $\mathbb{R}$ containing $\Omega$. An eigenvalue optimization problem of the form (1.4) is typically non-convex excluding the very special affine case $\mathscr{A}(\omega)=A_{1}+\omega A_{2}$.

Recently we have proposed general algorithms (Mengi et al. (2014); Kangal et al. (2018)) that are in many cases effective in solving non-convex eigenvalue optimization problems of the form (1.4) globally. The former of these in Mengi et al. (2014), an adaptation of the algorithm in Breiman \& Cutler (1993) for eigenvalue optimization, employs piece-wise quadratic functions to model the objective eigenvalue function, and is meant for small- to medium-scale problems. The latter in Kangal et al. (2018) introduces a subspace framework to deal with problems when the size of $\mathscr{A}(\omega)$ is large. It repeatedly projects $\mathscr{A}(\omega)$ to small subspaces, and minimizes the largest eigenvalue of the resulting projected matrix-valued function. Such subspace ideas have also been explored in special contexts such as convex semidefinite programs (Helmberg \& Rendl (2000); Helmberg et al. (2014)), and the computation of the pseudospectral abscissa (Kressner \& Vandereycken (2014); Meerbergen et al. (2017)). 


\subsection{Contributions}

Here we present the adaptations of the algorithms in Mengi et al. (2014); Kangal et al. (2018) for the solution of (1.3). This paves the way for efficient determination of whether a Hermitian pair $(A, B)$ is definite or not, as well as efficient computation of the distance $d_{\delta}(A, B)$ in (1.2) and a nearest definite pair $(\widetilde{A}, \widetilde{B})$ such that $\gamma(\widetilde{A}, \widetilde{B}) \geqslant \delta$. The adaptation of the algorithm in Mengi et al. (2014) is guaranteed to converge to the global minimizer of (1.3), and performs well in practice on small- to medium-scale problems. The subspace framework in Kangal et al. (2018) extends the range of applicability to quite large Hermitian matrix pairs.

Important contributions of this work are on the theoretical side. Global convergence of the algorithms has already been established; the subspace framework in Kangal et al. (2018) is globally convergent for the family of optimization problems (1.4) provided the projected problems are solved globally, whereas the algorithm in Mengi et al. (2014) is globally convergent for problems of the form (1.4) provided a global lower bound is known on $\lambda_{\text {max }}^{\prime \prime}(\mathscr{A}(\omega))$ over all $\omega$ where $\lambda_{\max }(\mathscr{A}(\omega))$ is differentiable. We are also quite informed about the rate-of-convergences of these algorithms in the smooth case when $\lambda_{\max }\left(\mathscr{A}\left(\omega_{*}\right)\right)$ is simple at a converged global minimizer $\omega_{*}$; the subspace framework converges at a superlinear rate (see Kangal et al. (2018); Kressner et al. (2018)) both in theory and in practice, while we observe that the algorithm in Mengi et al. (2014) converges at a linear rate (even though a formal proof is open, numerical experiments indicate a linear convergence convincingly). However, little is known about the rate-of-convergences in the presence of non-smoothness when $\lambda_{\max }\left(\mathscr{A}\left(\omega_{*}\right)\right)$ is not simple. In this work, we analyze the rate-of-convergences of the algorithms in Mengi et al. (2014) and in Kangal et al. (2018); Kressner et al. (2018) on the problems of the form (1.4) in the non-smooth setting, when $\lambda_{\max }\left(\mathscr{A}\left(\omega_{*}\right)\right)$ is multiple. We prove, in this non-smooth case and under generic assumptions, that if the maximum of the errors of the last two iterates of the algorithm in Mengi et al. (2014) is $h$, the error of the next iterate is $O\left(h^{2}\right)$. We also generalize the subspace framework in Kangal et al. (2018) to cope with the non-smooth setting, and show rigorously that the iterates of the proposed generalized framework converge at a quadratic rate in the presence of non-smoothness generically.

\subsection{Outline}

We present our work in the following order. Background on definite pairs and on the distance $d_{\delta}(A, B)$ are summarized in Section 2; in particular the links between these concepts, the eigenvalue optimization problem (1.3) and the inner numerical radius of $A+\mathrm{i} B$ have been discussed. The crucial task is the solution of (1.3), equivalently the computation of the inner numerical radius of $A+\mathrm{i} B$. In Section 3, the algorithm in Mengi et al. (2014) based on piece-wise quadratic support functions is presented in the general scope of (1.4) pointing out how it can be adapted for (1.3) to compute the inner numerical radii for small to medium size matrices. The remarkable contribution is a rate-of-convergence analysis in Section 3.2 for the piece-wise quadratic support based algorithm in the non-smooth setting when $\lambda_{\max }(\mathscr{A}(\omega))$ is multiple and not differentiable at a converged global minimizer. The analysis indicates a rapid convergence, surprisingly faster than the smooth case when $\lambda_{\max }(\mathscr{A}(\omega))$ is simple at the minimizer. This is followed by Section 4 which is devoted to the subspace framework to deal with (1.4) when the Hermitian matrices $A_{1}, \ldots, A_{\kappa}$ are large; the proposed framework generalizes the basic one in Kangal et al. (2018), Kressner et al. (2018) taking into account also the possible non-smoothness at the optimal point. It is in particular applicable to solve (1.3) and to compute the inner numerical radius of $A+\mathrm{i} B$ for large $A, B$. In Section 4.3, we establish a quadratic rate-of-convergence of the proposed subspace framework formally in the non-smooth case, which was left open by the previous works. Numerical examples at the ends of Sections 3 and 4 illustrate the efficiency of the algorithms, and confirm 
that the rate-of-convergences established in theory are realized in practice.

\section{Background on Definite Pairs and Nearest Definite Pairs}

\subsection{Generalized Eigenvalue Problems involving Definite Hermitian Pairs}

As argued in the opening, the significance of the definiteness of $(A, B)$ is that, it turns out there exists a related Hermitian pair $(\widetilde{A}, \widetilde{B})$ such that $\lambda_{\min }(\widetilde{B})=\gamma(A, B)$. Such a $(\widetilde{A}, \widetilde{B})$ can be obtained by applying rotations to the field of values as explained next.

It can be readily seen that $F\left(e^{-\mathrm{i} \theta}(A+\mathrm{i} B)\right)=e^{-\mathrm{i} \theta} F(A+\mathrm{i} B)$, so $F\left(e^{-\mathrm{i} \theta}(A+\mathrm{i} B)\right)$ is obtained from $F(A+\mathrm{i} B)$ by a rotation in the complex plane in the clock-wise direction by the angle of $\theta$. Thus, setting $(A(\theta), B(\theta))$ to the Hermitian pair satisfying $A(\theta)+\mathrm{i} B(\theta)=e^{-\mathrm{i} \theta}(A+\mathrm{i} B)$, that is

$$
A(\theta):=A \cos \theta+B \sin \theta \quad \text { and } B(\theta):=-A \sin \theta+B \cos \theta,
$$

we must have $\gamma(A, B)=\gamma(A(\theta), B(\theta)) \geqslant \lambda_{\min }(B(\theta))$ for all $\theta \in[0,2 \pi]$. Furthermore, it can be shown that $\gamma(A, B)=\gamma(A(\varphi), B(\varphi))=\lambda_{\min }(B(\varphi))$ for $\varphi:=-\pi / 2+\phi$ where $\phi \in[0,2 \pi]$ is such that $\gamma(A, B) e^{\mathrm{i} \phi}=$ $\arg \min _{z \in F(A+\mathrm{i} B)}|z|$. Let us also remark that there is an explicit one-to-one correspondence between the eigenvalues of $(A, B)$ and $(A(\varphi), B(\varphi))$; for details, we refer to Cheng \& Higham (1999); Stewart (1979).

The optimal $\varphi:=\phi-\pi / 2$ in the discussions above is defined in terms of $\phi \in[0,2 \pi]$ satisfying $\gamma(A, B) e^{\mathrm{i} \phi}=\arg \min _{z \in F(A+\mathrm{i} B)}|z|$. Such a $\phi$ can be obtained by solving the optimization problem in (1.3); indeed $\phi=\theta_{*}+\pi$ for

$$
\theta_{*}:=\underset{\theta \in[0,2 \pi]}{\arg \min } \lambda_{\max }(A(\theta):=A \cos \theta+B \sin \theta),
$$

(Cheng \& Higham (1999)), which in turn implies $\varphi=\theta_{*}+\pi / 2$.

In summary, the Hermitian matrices $\widetilde{A}:=A(\varphi)$ and $\widetilde{B}:=B(\varphi)$ for the angle $\varphi=\theta_{*}+\pi / 2$ satisfy $\widetilde{A}+\mathrm{i} \widetilde{B}=e^{-\mathrm{i} \varphi}(A+\mathrm{i} B)$ with $\lambda_{\min }(\widetilde{B})=\gamma(A, B)$.

\subsection{Inner Numerical Radius}

In geometric terms, regardless of $(A, B)$ is definite or not, the globally smallest value of (1.3) in absolute value corresponds to the modulus of the point on the boundary of $F(A+\mathrm{i} B)$ closest to the origin. Formally,

$$
\begin{aligned}
\zeta(A, B) & :=\min \{|z| \mid z \text { is on the boundary of } F(A+\mathrm{i} B)\} \\
& =\left|\min _{\theta \in[0,2 \pi]} \lambda_{\max }(A \cos \theta+B \sin \theta)\right| .
\end{aligned}
$$

The quantity $\zeta(A, B)$ is called the inner numerical radius of $A+\mathrm{i} B$.

The definiteness of a Hermitian pair $(A, B)$ is equivalent to whether $\zeta(A, B)=\gamma(A, B)$, and this can be determined by the sign of $\lambda_{*}:=\min _{\theta \in[0,2 \pi]} \lambda_{\max }(A \cos \theta+B \sin \theta)$. In particular, the pair $(A, B)$ is definite if and only if $\lambda_{*}<0$ (Cheng \& Higham (1999)).

\subsection{Nearest Definite Pairs}

For a prescribed real number $\delta>0$, we consider $d_{\delta}(A, B)$ defined as in (1.2). It is shown in Cheng $\&$ Higham (1999) that

$$
d_{\delta}(A, B)=\max \left\{\delta+\lambda_{*}, 0\right\}
$$


where $\lambda_{*}:=\min _{\theta \in[0,2 \pi]} \lambda_{\max }(A \cos \theta+B \sin \theta)$. Moreover, letting $\theta_{*}$ as in (2.1), and $A \cos \theta_{*}+B \sin \theta_{*}=$ $Q \operatorname{diag}\left(\lambda_{i}\right) Q^{*}$ with $\lambda_{n} \leqslant \cdots \leqslant \lambda_{1}$ be a spectral decomposition, an optimal solution $\left(\Delta A_{*}, \Delta B_{*}\right)$ for (1.2) is given by

$$
\Delta A_{*}=\cos \theta_{*} Q \operatorname{diag}\left(\min \left\{-\delta-\lambda_{i}, 0\right\}\right) Q^{*}, \quad \Delta B_{*}=\sin \theta_{*} Q \operatorname{diag}\left(\min \left\{-\delta-\lambda_{i}, 0\right\}\right) Q^{*} .
$$

It is also deduced in Cheng \& Higham (1999) that

$$
\gamma\left(A+\Delta A_{*}, B+\Delta B_{*}\right) e^{\mathrm{i} \phi}=\underset{z \in F\left(A+\Delta A_{*}+\mathrm{i}\left(B+\Delta B_{*}\right)\right)}{\arg \min }|z|
$$

for $\phi=\theta_{*}+\pi$. Now it follows from the discussions in Section 2.1 that, letting $\widetilde{A}, \widetilde{B}$ such that $\widetilde{A}+\mathrm{i} \widetilde{B}=$ $e^{-\mathrm{i} \varphi}\left(A+\Delta A_{*}+\mathrm{i}\left(B+\Delta B_{*}\right)\right)$ for $\varphi=\theta_{*}+\pi / 2$, we have $\lambda_{\min }(\widetilde{B})=\gamma\left(A+\Delta A_{*}, B+\Delta B_{*}\right) \geqslant \delta$.

Finally, it is argued in Cheng \& Higham (1999) that the most challenging part in the determination of the optimal angle $\varphi$, as well as the optimal perturbations $\Delta A_{*}, \Delta B_{*}$ is the solution of (1.3) globally. In the next section we focus on the solution of these non-convex eigenvalue optimization problems.

\section{Computation of Inner Numerical Radius and Beyond}

The problem at hand can be expressed as $\zeta(A, B)=\left|\min _{\theta \in[0,2 \pi]} f(\theta)\right|$, where $f(\theta)=\lambda_{\max }(A \cos \theta+$ $B \sin \theta)$. It is possible to devise a quadratically convergent level-set method to minimize $f(\theta)$ globally analogous to the one to compute the numerical radius described in Mengi \& Overton (2005), and an extension of the Boyd-Balakrishnan algorithm to compute the $\mathscr{H}_{\infty}$-norm (Boyd \& Balakrishnan (1990)). This is discussed in detail in Kangal \& Mengi (2018), a longer version of this text. The computational bottleneck is that the method needs to extract all eigenvalues of unit modulus of a $2 n \times 2 n$ matrix pencil in order to determine the $\alpha$-level set of $f(\theta)$ (i.e., the set $\{\theta \mid f(\theta)=\alpha\}$ ) for a given $\alpha$; see Theorem 3.1 in Mengi \& Overton (2005), Theorem 2 in He \& Watson (1997).

The support based algorithm discussed next requires the computation of only the largest eigenvalues of $A(\theta):=A \cos \theta+B \sin \theta$ several times. Unlike the level-set method whose rate-of-convergence is diminished to a linear convergence in the non-smooth setting when $\lambda_{\max }\left(A\left(\theta_{*}\right)\right)$ is multiple at the minimizer $\theta_{*}$ defined in (2.1), the support based algorithm converges rapidly in the same non-smooth setting, which is stated precisely and proved formally in Section 3.2.

\subsection{Support Based Algorithm}

The algorithm that we employ in this section for computing the inner numerical radius is borrowed from Mengi et al. (2014). Throughout the section, we consider the general setting of (1.4). The algorithm that we discuss here to solve (1.4) globally is based on the boundedness of the second derivatives of the objective eigenvalue function. It replaces the eigenvalue functions with piece-wise quadratic support functions that underestimate $\lambda_{\max }(\mathscr{A}(\omega))$ globally.

We first introduce formally the quadratic support functions, which are the main ingredients of the algorithm built on the analytical properties of $\lambda_{\max }(\mathscr{A}(\omega))$. The next result states the analytical properties that are relevant to the derivation (see e.g. Rellich (1969); Kato (1995); Lancaster (1964)).

Lemma 3.1 Let $\mathscr{A}(\omega): \mathbb{R} \rightarrow \mathbb{C}^{n \times n}$ be a Hermitian matrix-valued function as in (1.4) and $\widetilde{\omega} \in \mathbb{R}$. The following hold:

(i) The eigenvalues of $\mathscr{A}(\omega)$ can be permuted in a way so that the permuted eigenvalues $\widetilde{\lambda}_{1}(\omega), \ldots$, $\widetilde{\lambda}_{n}(\omega)$ are real analytic functions of $\omega$ everywhere. 
(ii) For a given $p \in \mathbb{R}$, letting $\phi(\alpha):=\lambda_{\max }(\mathscr{A}(\widetilde{\omega}+\alpha p))$, the left-hand derivative $\phi_{-}^{\prime}(\alpha)$ and the right-hand derivative $\phi_{+}^{\prime}(\alpha)$ of $\phi(\alpha)$ exist everywhere, furthermore they satisfy $\phi_{+}^{\prime}(\alpha) \geqslant \phi_{-}^{\prime}(\alpha)$ at all $\alpha \in \mathbb{R}$.

(iii) If $\lambda_{\max }(\mathscr{A}(\widetilde{\omega}))$ is simple, $\lambda_{\max }(\mathscr{A}(\omega))$ is real analytic at $\widetilde{\omega}$ with the derivatives

$$
\begin{aligned}
\frac{d \lambda_{\max }(\mathscr{A}(\widetilde{\omega}))}{d \omega}= & v^{*} \frac{d \mathscr{A}(\widetilde{\omega})}{d \omega} v, \quad \text { and } \\
\frac{d^{2} \lambda_{\max }(\mathscr{A}(\widetilde{\omega}))}{d \omega^{2}}= & v^{*} \frac{d^{2} \mathscr{A}(\widetilde{\omega})}{d \omega^{2}} v \\
& \quad+2 \sum_{k=2}^{n} \frac{1}{\lambda_{\max }(\mathscr{A}(\widetilde{\omega}))-\lambda_{k}(\mathscr{A}(\widetilde{\omega}))}\left|v_{k}^{*} \frac{d \mathscr{A}(\widetilde{\omega})}{d \omega} v\right|^{2},
\end{aligned}
$$

where $\lambda_{k}(\mathscr{A}(\widetilde{\omega}))$ denotes the $k$ th largest eigenvalue of $\mathscr{A}(\widetilde{\omega})$, and $v, v_{k}$ are unit eigenvectors corresponding to $\lambda_{\max }(\mathscr{A}(\widetilde{\omega})), \lambda_{k}(\mathscr{A}(\widetilde{\omega}))$, respectively, for $k=2 \ldots, n$.

In the next result we present the quadratic support functions. The proof of the fact that these functions are global under-estimators for the largest eigenvalue functions follows from part (ii) of Lemma 3.1. We omit the proof because of its similarity to the proof of Theorem 2.2 in Mengi (2017), the analogous result that constructs upper support functions for smallest eigenvalue functions.

THEOREM 3.1 (Quadratic Lower Support Functions) Suppose $\lambda_{\max }(\mathscr{A}(\omega))$ is simple at $\omega^{(k)} \in \Omega$. Additionally, suppose $\gamma$ satisfies $\lambda_{\max }^{\prime \prime}(\mathscr{A}(\omega)) \geqslant \gamma$ for all $\omega \in \Omega$ such that $\lambda_{\max }(\mathscr{A}(\omega))$ is simple. Then, we have

$$
\lambda_{\max }(\mathscr{A}(\omega)) \geqslant q_{k}(\omega):=\lambda_{k}+\lambda_{k}^{\prime}\left(\omega-\omega^{(k)}\right)+\frac{\gamma}{2}\left(\omega-\omega^{(k)}\right)^{2} \quad \forall \omega \in \Omega
$$

where $\lambda_{k}:=\lambda_{\max }\left(\mathscr{A}\left(\omega^{(k)}\right)\right), \lambda_{k}^{\prime}:=\lambda_{\max }^{\prime}\left(\mathscr{A}\left(\omega^{(k)}\right)\right)$.

We call $q_{k}(\omega)$ as in (3.2) the quadratic support function about $\omega^{(k)}$. Such a quadratic support function is defined in terms of a lower bound $\gamma$ for the second derivatives of the eigenvalue function, which can occasionally be obtained from expression (3.1) for the second derivatives of $\lambda_{\max }(\mathscr{A}(\omega))$. Since the summation term at the bottom row of (3.1) is non-negative, we must have

$$
\frac{d^{2} \lambda_{\max }(\mathscr{A}(\omega))}{d \omega^{2}} \geqslant v^{*} \frac{d^{2} \mathscr{A}(\omega)}{d \omega^{2}} v \geqslant-\left\|\frac{d^{2} \mathscr{A}(\omega)}{d \omega^{2}}\right\|_{2}
$$

at all $\omega$ where $\lambda_{\max }(\mathscr{A}(\omega))$ is simple. Hence $\gamma=-\max _{\omega \in \Omega}\left\|d^{2} \mathscr{A}(\omega) / d \omega^{2}\right\|_{2}$ is a theoretically sound choice.

In the case of computing the inner numerical radius of $A+\mathrm{i} B$ for a given Hermitian pair $(A, B)$, we minimize $\lambda_{\max }(A(\theta):=A \cos \theta+B \sin \theta)$ over $\theta$. By the arguments of the previous paragraph

$$
\frac{d^{2} \lambda_{\max }(A(\theta))}{d \theta^{2}} \geqslant-\|A \cos \theta+B \sin \theta\|_{2} \geqslant-\|A\|_{2}-\|B\|_{2},
$$

so we set $\gamma=-\|A\|_{2}-\|B\|_{2}$.

Finally we present the algorithm based on these support functions. It generates a sequence $\left\{\omega^{(k)}\right\}$ of estimates for a global minimizer of $\lambda_{\max }(\mathscr{A}(\omega))$, a sequence $\left\{\ell^{(k)}\right\}$ of lower bounds for the globally smallest value of $\lambda_{\max }(\mathscr{A}(\omega))$ and a sequence $\left\{\bar{q}_{k}(\omega)\right\}$ of piece-wise quadratic model functions for 
$\lambda_{\max }(\mathscr{A}(\omega))$. At the $k$ th iteration, the point $\omega^{(k+1)}$ is set equal to a global minimizer of the piecewise quadratic model function $\bar{q}_{k}(\omega):=\max _{j=0, \ldots, k} q_{j}(\omega)$. This is followed by the construction of the quadratic support function $q_{k+1}(\omega)$ about $\omega^{(k+1)}$, and inclusion of $q_{k+1}(\omega)$ in the piece-wise quadratic model function. A formal description is given in Algorithm 1.

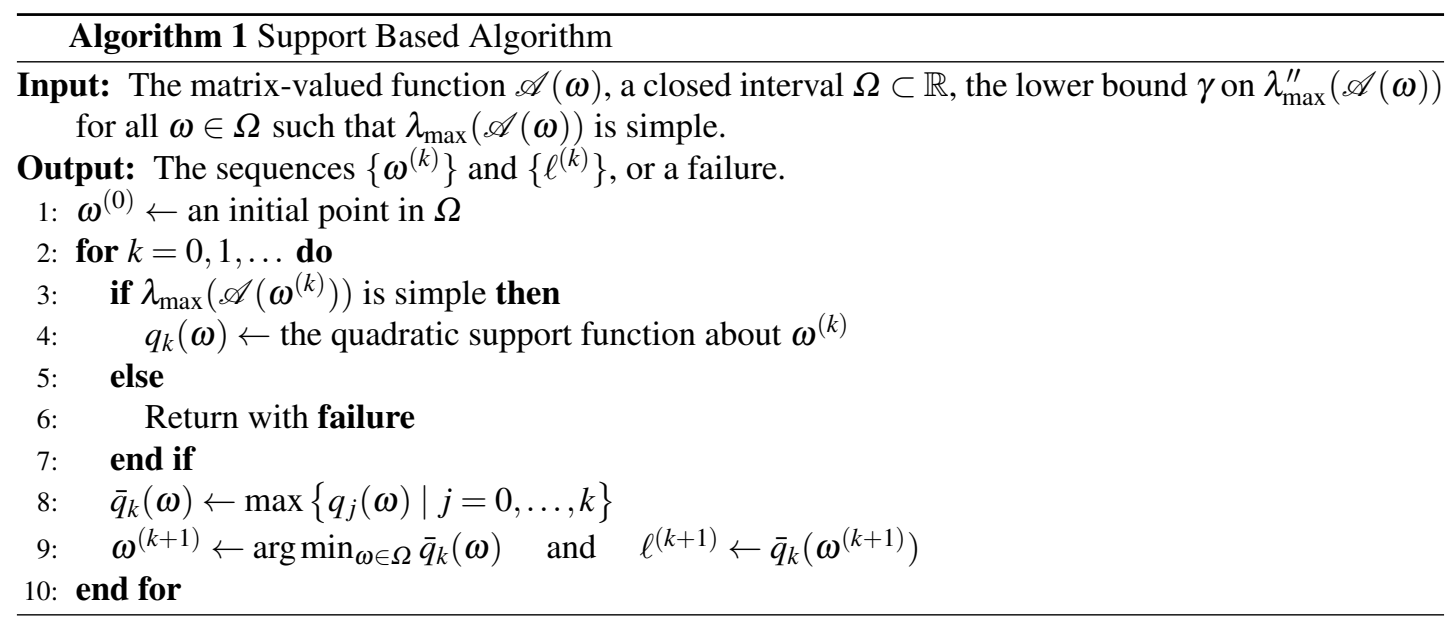

REMARK 3.1 (Generalization of Algorithm 1 to deal with non-differentiability of $\lambda_{\max }(\mathscr{A}(\omega))$ at $\omega^{(k)}$ ) The algorithm as stated above terminates with a failure if $\lambda_{\max }\left(\mathscr{A}\left(\omega^{(k)}\right)\right)$ is not simple, as in this situation $\lambda_{\max }^{\prime}(\mathscr{A}(\omega))$ may not be differentiable at $\omega^{(k)}$ and $q_{k}(\omega)$ as in (3.2) may not be well-defined.

The support functions and the algorithm can be generalized to deal with the case when $\lambda_{\max }(\mathscr{A}(\omega))$ is multiple at $\omega^{(k)}$. Theorem 3.1, in particular (3.2), hold even when $\lambda\left(\mathscr{A}\left(\omega^{(k)}\right)\right)$ is not simple provided that in the definition of $q_{k}(\omega)$ the vector $\lambda_{k}^{\prime}$ is now any vector that belongs to the Clarke generalized derivative $\partial \lambda_{\max }\left(\mathscr{A}\left(\omega^{(k)}\right)\right)$. Thus, in this non-smooth setting, one could define Algorithm 1 the same as above but based on this generalized definition of $q_{k}(\omega)$. Recall that the generalized Clarke derivative of $f(\omega)$ at $\widetilde{\omega}$, for a univariate function $f$ that is differentiable almost everywhere excluding a set $\Gamma$ of measure zero, is given by (Clarke (1990))

$$
\partial f(\widetilde{\omega}):=\operatorname{Co}\left\{\lim _{k \rightarrow \infty} f^{\prime}\left(\widetilde{\omega}^{(k)}\right) \mid \widetilde{\omega}^{(k)} \rightarrow \widetilde{\omega}, \widetilde{\omega}^{(k)} \notin \Gamma \forall k\right\}
$$

with $\operatorname{Co}(S)$ denoting the convex hull of the set $S$.

By part (i) of Lemma 3.1, any point where $\lambda_{\max }(\mathscr{A}(\omega))$ is not differentiable is isolated, so the set of such points of non-differentiability over $\Omega$ is of measure zero. In practice we employ Algorithm 1 by setting $\lambda_{k}$ as the computed largest eigenvalue of $\mathscr{A}\left(\omega^{(k)}\right)$ after rounding errors; we have not encountered an example where this strategy causes numerical difficulties. However, if it happens that $\left\{\omega^{(k)}\right\}$ approaches a point $\omega_{*}$ where $\lambda_{\max }(\mathscr{A}(\omega))$ is not differentiable, the rate-of-convergence of $\left\{\omega^{(k)}\right\}$ is affected by the non-differentiability at $\omega_{*}$. This is the main issue studied in Section 3.2 below.

AsSUMPTION 3.2 Throughout the rest of this section, it is always assumed that $\lambda_{\max }\left(\mathscr{A}\left(\omega^{(k)}\right)\right)$ is simple for all $k$. However, it is straightforward to verify that the global convergence and rate-of-convergence results below extend to the generalized version of Algorithm 1 described in Remark 3.1. 
The next result draws global convergence conclusions regarding the sequences $\left\{\omega^{(k)}\right\}$ and $\left\{\ell^{(k)}\right\}$ by Algorithm 1. This has been proven in Theorem 8.1 in Mengi et al. (2014).

THEOREM 3.3 Every convergent subsequence of the sequence $\left\{\omega^{(k)}\right\}$ by Algorithm 1 converges to a global minimizer of $\lambda_{\max }(\mathscr{A}(\omega))$ over $\omega \in \Omega$. Furthermore,

$$
\lim _{k \rightarrow \infty} \ell^{(k)}=\min _{\omega \in \Omega} \lambda_{\max }(\mathscr{A}(\omega)) .
$$

The next result immediately follows from Theorem 3.3.

Theorem 3.4 Suppose that $\lambda_{\max }(\mathscr{A}(\omega))$ has a unique global minimizer over all $\omega \in \Omega$, say at $\omega_{*}$. Then the sequence $\left\{\omega^{(k)}\right\}$ by Algorithm 1 converges to $\omega_{*}$.

\subsection{Rate-of-Convergence of the Support Based Algorithm}

In numerous numerical experiments, we observe that Algorithm 1 converges at a linear rate in the smooth case if $\lambda_{\max }(\mathscr{A}(\omega))$ is simple at its global minimizers, but a formal proof of this observation is open at the moment.

Here we turn our attention to the non-smooth case, in particular provide a formal rate-of-convergence analysis in this case. Remarkably the presence of non-smoothness accelerates Algorithm 1. This is quite a contrast to the level-set method discussed in Kangal \& Mengi (2018), whose quadratic convergence in the smooth case is hindered and limited to a linear convergence by the existence of non-smoothness.

Throughout the rest we assume $\lambda_{\max }(\mathscr{A}(\omega))$ has a unique global minimizer at $\omega_{*}$ that is strictly in the interior of $\Omega$, and $\lambda_{\max }\left(\mathscr{A}\left(\omega_{*}\right)\right)$ is multiple with $0 \in \operatorname{Int} \partial \lambda_{\max }\left(\mathscr{A}\left(\omega_{*}\right)\right)$, where $\partial \lambda_{\max }\left(\mathscr{A}\left(\omega_{*}\right)\right)$ is the generalized Clarke derivative of $\lambda_{\max }(\mathscr{A}(\omega))$ at $\omega_{*}$ (see (3.3) for the definition of the generalized Clarke derivative), Int $\partial \lambda_{\max }\left(\mathscr{A}\left(\omega_{*}\right)\right)$ is the interior of $\partial \lambda_{\max }\left(\mathscr{A}\left(\omega_{*}\right)\right)$.

By part (i) of Lemma 3.1, the eigenvalue function $\lambda_{\max }(\mathscr{A}(\omega))$ is continuous and piece-wise real analytic at $\omega_{*}$, indeed there exists an open interval $\mathscr{I} \subseteq$ Int $\Omega$ containing $\omega_{*}$ and two real analytic eigenvalue functions $\widetilde{\lambda}_{1}(\mathscr{A}(\omega)), \widetilde{\lambda}_{2}(\mathscr{A}(\omega))$ such that

$$
\lambda_{\max }(\mathscr{A}(\omega))= \begin{cases}\tilde{\lambda}_{1}(\mathscr{A}(\omega)) & \omega \in \mathscr{I}, \omega \geqslant \omega_{*}, \\ \tilde{\lambda}_{2}(\mathscr{A}(\omega)) & \omega \in \mathscr{I}, \omega \leqslant \omega_{*} .\end{cases}
$$

Now we cannot have $\tilde{\lambda}_{1}^{\prime}\left(\mathscr{A}\left(\omega_{*}\right)\right)<0$ as this would contradict the fact that $\omega_{*}$ is a minimizer of $\lambda_{\max }\left(\mathscr{A}(\omega)\right.$. Furthermore, $\tilde{\lambda}_{1}^{\prime}\left(\mathscr{A}\left(\omega_{*}\right)\right) \neq 0$ due to the assumption $0 \in \operatorname{Int} \partial \lambda_{\max }\left(\mathscr{A}\left(\omega_{*}\right)\right)$. Hence, we must have $\lambda_{*,+}^{\prime}:=\tilde{\lambda}_{1}^{\prime}\left(\mathscr{A}\left(\omega_{*}\right)\right)>0$. By an analogous argument $\lambda_{*,-}^{\prime}:=\tilde{\lambda}_{2}^{\prime}\left(\mathscr{A}\left(\omega_{*}\right)\right)<0$. The quantities $\lambda_{*,+}^{\prime}$ and $\lambda_{*,-}^{\prime}$ correspond to the right-hand and left-hand derivatives, respectively, of $\lambda_{\max }(\mathscr{A}(\omega))$ at $\omega_{*}$. In what follows, we also use the notations $\lambda_{*,+}^{\prime \prime}:=\tilde{\lambda}_{1}^{\prime \prime}\left(\mathscr{A}\left(\omega_{*}\right)\right), \lambda_{*,-}^{\prime \prime}:=\tilde{\lambda}_{2}^{\prime \prime}\left(\mathscr{A}\left(\omega_{*}\right)\right)$, which correspond to one-sided second derivatives of $\lambda_{\max }(\mathscr{A}(\omega))$ at $\omega_{*}$, and $\lambda_{*}:=\lambda_{\max }\left(\mathscr{A}\left(\omega_{*}\right)\right)$. Additionally, the short-hands $\lambda_{k}, \lambda_{k}^{\prime}, \lambda_{k}^{\prime \prime}$ represent $\lambda_{\max }\left(\mathscr{A}\left(\omega^{(k)}\right)\right), \lambda_{\max }^{\prime}\left(\mathscr{A}\left(\omega^{(k)}\right)\right), \lambda_{\max }^{\prime \prime}\left(\mathscr{A}\left(\omega^{(k)}\right)\right)$ at an iterate $\omega^{(k)}$ of Algorithm 1. Finally, the lower bound $\gamma$ for the second derivatives of $\lambda_{\max }(\mathscr{A}(\omega))$ is assumed to be negative throughout this subsection without loss of generality.

The next result characterizes the global minimizer $\omega^{(k+1)}$ of $\bar{q}_{k}(\omega)$ for large $k$. This point always turns out to be the intersection point of the two quadratic support functions about the iterates that are closest to $\omega_{*}$ among the iterates on the left-hand and on the right-hand side of $\omega_{*}$. Moreover, if the distance between the iterates about which these two support functions are constructed is $h$, then $\omega^{(k+1)}$ is located at a distance of $O\left(h^{2}\right)$ to $\omega_{*}$. 
Lemma 3.2 (Minimizers of the Support Functions) Suppose $\omega_{*}$ is the unique global minimizer of $\lambda_{\max }(\mathscr{A}(\omega))$ over $\omega \in \Omega$ such that $\omega_{*} \in \operatorname{Int} \Omega$, and the eigenvalue $\lambda_{\max }\left(\mathscr{A}\left(\omega_{*}\right)\right)$ is multiple, $0 \in$ Int $\partial \lambda_{\max }\left(\mathscr{A}\left(\omega_{*}\right)\right), \gamma<0$. Additionally, suppose that the sequence $\left\{\omega^{(k)}\right\}$ by Algorithm 1 is such that $\omega^{(k)} \neq \omega_{*}$ for all $k$. Then $\left\{\omega^{(k)}\right\}$ satisfies the following for all $k$ large enough:

(i) The point $\omega^{(k+1)}$ is the intersection point of $q_{\ell(k)}(\omega)$ and $q_{r(k)}(\omega)$, where $\ell(k), r(k) \in\{0, \ldots, k\}$ are given by

$$
\begin{aligned}
& \ell(k):=\arg \min \left\{\omega_{*}-\omega^{(j)} \mid j \in\{0, \ldots, k\} \text { s.t. } \omega_{*}>\omega^{(j)}\right\} \quad \text { and } \\
& r(k):=\arg \min \left\{\omega^{(j)}-\omega_{*} \mid j \in\{0, \ldots, k\} \text { s.t. } \omega_{*}<\omega^{(j)}\right\} .
\end{aligned}
$$

(ii) Letting $h:=\max \left\{\omega_{*}-\omega^{(\ell(k))}, \omega^{(r(k))}-\omega_{*}\right\}$, we have

$$
\omega^{(k+1)}=\alpha \cdot \omega_{*}+\beta \cdot\left(\frac{\omega^{(\ell(k))}+\omega^{(r(k))}}{2}\right)+O\left(h^{2}\right),
$$

where $\alpha, \beta \in \mathbb{R}^{+}$are such that $\alpha+\beta=1$ and $\beta=\Theta(h)$.

(iii) Furthermore, $\left|\omega^{(k+1)}-\omega_{*}\right|=O\left(h^{2}\right)$.

Proof. (i) The real analyticity of $\widetilde{\lambda}_{1}(\mathscr{A}(\omega)), \widetilde{\lambda}_{2}(\mathscr{A}(\omega))$ imply that these eigenvalue functions are continuously differentiable. Furthermore, by (3.4), we have $\lambda_{\max }^{\prime}(\mathscr{A}(\omega))=\tilde{\lambda}_{1}^{\prime}(\mathscr{A}(\omega))$ for all $\omega \in \mathscr{I}$ such that $\omega>\omega_{*}$, and $\lambda_{\max }^{\prime}(\mathscr{A}(\omega))=\tilde{\lambda}_{2}^{\prime}(\mathscr{A}(\omega))$ for all $\omega \in \mathscr{I}$ such that $\omega<\omega_{*}$. Hence, there exists an interval $\widetilde{\mathscr{I}}:=\left(\omega_{*}-\delta, \omega_{*}+\delta\right) \subseteq \mathscr{I}$ for some $\delta>0$ such that

$$
\lambda_{\max }^{\prime}(\mathscr{A}(\omega)) \geqslant \frac{\lambda_{*,+}^{\prime}}{2} \quad \forall \omega \in\left(\omega_{*}, \omega_{*}+\delta\right) \quad \text { and } \quad \lambda_{\max }^{\prime}(\mathscr{A}(\omega)) \leqslant \frac{\lambda_{*,-}^{\prime}}{2} \quad \forall \omega \in\left(\omega_{*}-\delta, \omega_{*}\right) .
$$

We can choose $\delta$ as small as we wish. In particular, in the subsequent arguments, we assume $\delta \leqslant$ $\min \left\{\left(\lambda_{*,-}^{\prime}\right) /(4 \gamma),-\left(\lambda_{*,+}^{\prime}\right) /(4 \gamma)\right\}$ without loss of generality.

We first show that $\omega^{(k+1)} \in\left[\omega^{(\ell(k))}, \omega^{(r(k))}\right]$. Theorem 3.4 asserts that $\omega^{(k)} \rightarrow \omega_{*}$ as $k \rightarrow \infty$, so for all $k$ large enough $\omega^{(k)} \in \widetilde{\mathscr{I}}$. Letting $\omega_{r}^{(k)}:=\omega^{(r(k))}$, as the sequence $\left\{\omega_{r}^{(k)}\right\}$ is monotonically decreasing and bounded below by $\omega_{*}$, it must converge to a point, say $\omega_{*, r} \geqslant \omega_{*}$. Similarly, letting $\omega_{\ell}^{(k)}:=\omega^{(\ell(k))}$, the sequence $\left\{\omega_{\ell}^{(k)}\right\}$ must converge to a point $\omega_{*, \ell} \leqslant \omega_{*}$. The conditions $\omega_{*, \ell} \neq \omega_{*}$ and $\omega_{*, r} \neq \omega_{*}$ contradict the convergence of $\left\{\omega^{(k)}\right\}$ to $\omega_{*}$. Hence, at least one of $\left\{\omega_{r}^{(k)}\right\}$ and $\left\{\omega_{\ell}^{(k)}\right\}$ must converge to $\omega_{*}$. Without loss of generality suppose this is the case with $\left\{\omega_{\ell}^{(k)}\right\}$. As a result, we also deduce $\omega^{(\ell(k))} \in \widetilde{I}$ for all large $k$. For such a large $k$, we have

$$
q_{\ell(k)}^{\prime}\left(\omega^{(\ell(k))}\right)=\lambda_{\max }^{\prime}\left(\mathscr{A}\left(\omega^{(\ell(k))}\right)\right) \leqslant \frac{\lambda_{*,-}^{\prime}}{2} .
$$

Additionally, the inequality $\delta \leqslant\left(\lambda_{*,-}^{\prime}\right) /(4 \gamma)$ implies

$$
q_{\ell(k)}^{\prime}(\omega)=\lambda_{\max }^{\prime}\left(\mathscr{A}\left(\omega^{(\ell(k))}\right)\right)+\gamma\left(\omega-\omega^{(\ell(k))}\right) \leqslant \frac{\lambda_{*,-}^{\prime}}{2}-\gamma \delta \leqslant \frac{\lambda_{*,-}^{\prime}}{4}
$$


for all $\omega \in\left(\omega_{*}-\delta, \omega^{(\ell(k))}\right)$. This means $q_{\ell(k)}(\omega)$ is decreasing on this interval. Notice also that $q_{\ell(k)}\left(\omega^{(\ell(k))}\right)=\lambda_{\max }\left(\mathscr{A}\left(\omega^{(\ell(k))}\right)\right)$, as well as $\lambda_{\max }(\mathscr{A}(\omega)) \geqslant \bar{q}_{k}(\omega) \geqslant q_{\ell(k)}(\omega)$ for all $\omega$, together imply

$$
\lambda_{\max }\left(\mathscr{A}\left(\omega^{(\ell(k))}\right)\right)=\bar{q}_{k}\left(\omega^{(\ell(k))}\right)=q_{\ell(k)}\left(\omega^{(\ell(k))}\right) .
$$

Hence, we deduce

$$
\bar{q}_{k}\left(\omega^{(\ell(k))}\right)=q_{\ell(k)}\left(\omega^{(\ell(k))}\right)<q_{\ell(k)}(\omega) \leqslant \bar{q}_{k}(\omega) \quad \forall \omega \in\left(\omega_{*}-\delta, \omega^{(\ell(k))}\right) .
$$

Consequently, $\omega^{(k+1)}$, the global minimizer of $\bar{q}_{k}(\omega)$, cannot lie in $\left(\omega_{*}-\delta, \omega^{(\ell(k))}\right)$, that is $\omega^{(k+1)} \in$ $\left[\omega^{(\ell(k))}, \omega_{*}+\delta\right)$.

If $\omega^{(r(k))} \notin \widetilde{\mathscr{I}}$, then $\omega^{(r(k))}-\omega_{*} \geqslant \delta$, so we must have $\omega^{(k+1)} \in\left[\omega^{(\ell(k))}, \omega_{*}+\delta\right) \subseteq\left[\omega^{(\ell(k))}, \omega^{(r(k))}\right]$ as desired. Otherwise, $\omega^{(r(k))} \in \widetilde{\mathscr{I}}$, and analogous arguments as in the previous paragraph apply to $q_{r(k)}(\omega)$ to conclude that $\omega^{(k+1)} \notin\left(\omega^{(r(k))}, \omega_{*}+\delta\right)$, which in turn implies $\omega^{(k+1)} \in\left[\omega^{(\ell(k))}, \omega^{(r(k))}\right]$.

As for the global minimizer of $\bar{q}_{k}(\omega)$ in $\left[\omega^{(\ell(k))}, \omega^{(r(k))}\right]$, we first note that

$$
\bar{q}_{k}(\omega)=\max \left\{q_{\ell(k)}(\omega), q_{r(k)}(\omega)\right\} \quad \forall \omega \in\left[\omega^{(\ell(k))}, \omega^{(r(k))}\right],
$$

as it turns out

(a) $q_{\ell(k)}(\omega) \geqslant q_{j}(\omega) \quad \forall \omega \geqslant \omega^{(\ell(k))}$ and for all $j \leqslant k$ such that $\omega^{(j)}<\omega_{*}$,

(b) $q_{r(k)}(\omega) \geqslant q_{j}(\omega) \quad \forall \omega \leqslant \omega^{(r(k))}$ and for all $j \leqslant k$ such that $\omega^{(j)}>\omega_{*}$.

To see (a), consider any $j$ such that $j \leqslant k$ and $\omega^{(j)}<\omega_{*}$ (implying also $\omega^{(j)} \leqslant \omega^{(\ell(k))}$ ). For such a $j$, we have $\lambda_{\ell(k)}=\lambda_{\max }\left(\mathscr{A}\left(\omega^{(\ell(k))}\right)\right) \geqslant q_{j}\left(\omega^{(\ell(k))}\right)$. Additionally, letting $\widetilde{\omega}_{1}<\widetilde{\omega}_{2}<\cdots<\widetilde{\omega}_{\kappa-1}$ be the points in $\left(\omega^{(j)}, \omega^{(\ell(k))}\right)$ where $\lambda_{\max }(\mathscr{A}(\omega))$ is not differentiable, as well as setting $\widetilde{\omega}_{0}:=\omega^{(j)}$ and $\widetilde{\omega}_{K}:=\omega^{(\ell(k))}$ (recalling also the notations $\lambda_{\ell(k)}^{\prime}=\lambda_{\max }^{\prime}\left(\mathscr{A}\left(\omega^{(\ell(k))}\right)\right), \lambda_{j}^{\prime}=\lambda_{\max }^{\prime}\left(\mathscr{A}\left(\omega^{(j)}\right)\right)$ ),

$$
\begin{aligned}
\lambda_{\ell(k)}^{\prime} & =\lambda_{j}^{\prime}+\sum_{\ell=0}^{\kappa-1} \int_{0}^{1} \lambda_{\max }^{\prime \prime}\left(\mathscr{A}\left(\widetilde{\omega}_{\ell}+t\left(\widetilde{\omega}_{\ell+1}-\widetilde{\omega}_{\ell}\right)\right)\right)\left(\widetilde{\omega}_{\ell+1}-\widetilde{\omega}_{\ell}\right) d t \\
& \geqslant \lambda_{j}^{\prime}+\sum_{\ell=0}^{\kappa-1} \int_{0}^{1} \gamma\left(\widetilde{\omega}_{\ell+1}-\widetilde{\omega}_{\ell}\right) d t=\lambda_{j}^{\prime}+\gamma\left(\omega^{(\ell(k))}-\omega^{(j)}\right)=q_{j}^{\prime}\left(\omega^{(\ell(k))}\right) .
\end{aligned}
$$

Now by expanding $q_{j}(\omega)$ around $\omega^{(\ell(k))}$ (instead of $\left.\omega^{(j)}\right)$, for all $\omega \geqslant \omega^{(\ell(k))}$, we obtain

$$
\begin{aligned}
q_{j}(\omega) & =q_{j}\left(\omega^{(\ell(k))}\right)+q_{j}^{\prime}\left(\omega^{(\ell(k))}\right)\left(\omega-\omega^{(\ell(k))}\right)+\gamma / 2\left(\omega-\omega^{(\ell(k))}\right)^{2} \\
& \leqslant \lambda_{\ell(k)}+\lambda_{\ell(k)}^{\prime}\left(\omega-\omega^{(\ell(k))}\right)+\gamma / 2\left(\omega-\omega^{(\ell(k))}\right)^{2}=q_{\ell(k)}(\omega)
\end{aligned}
$$

proving (a). An analogous argument shows (b).

Now the global minimum of $\bar{q}_{k}(\omega)$ over $\left[\omega^{(\ell(k))}, \omega^{(r(k))}\right]$ must be attained either at one of the end points $\omega^{(\ell(k))}$ or $\omega^{(r(k))}$, or at a point where $\bar{q}_{k}(\omega)$ is differentiable with derivative equal to zero, or at a point of non-differentiability for $\bar{q}_{k}(\omega)$, that is at the intersection point of $q_{\ell(k)}(\omega)$ and $q_{r(k)}(\omega)$. The minimum cannot be attained at the end points; for $\omega^{(\ell(k))}$, we have

$$
q_{\ell(k)}\left(\omega^{(\ell(k))}\right)=\lambda_{\max }\left(\mathscr{A}\left(\omega^{(\ell(k))}\right)\right)>\lambda_{\max }\left(\mathscr{A}\left(\omega_{*}\right)\right) \geqslant \min _{\omega \in \Omega} \bar{q}_{k}(\omega),
$$


where the first inequality is due to the uniqueness of the global minimizer $\omega_{*}$, the second inequality is due to $\lambda_{\max }(\mathscr{A}(\omega)) \geqslant \bar{q}_{k}(\omega)$ for all $\omega \in \Omega$. By replacing $\ell(k)$ with $r(k)$ in (3.7), we also deduce $\omega^{(r(k))}$ is not the global minimizer. A point $\widetilde{\omega}$ where $\bar{q}_{k}(\omega)$ is differentiable with derivative equal to zero can also not be the global minimizer, because $\bar{q}_{k}^{\prime \prime}(\widetilde{\omega})=\gamma<0$. Hence, the minimum must be attained at the intersection point of $q_{\ell(k)}(\omega)$ and $q_{r(k)}(\omega)$.

(ii) It follows from part (i) that $\omega^{(k+1)}$ satisfies $q_{\ell(k)}\left(\omega^{(k+1)}\right)=q_{r(k)}\left(\omega^{(k+1)}\right)$. Now, recalling $\lambda_{\ell(k)}=$ $\lambda_{\max }\left(\mathscr{A}\left(\omega^{(\ell(k))}\right)\right), \lambda_{r(k)}=\lambda_{\max }\left(\mathscr{A}\left(\omega^{(r(k))}\right)\right), \lambda_{\ell(k)}^{\prime}=\lambda_{\max }^{\prime}\left(\mathscr{A}\left(\omega^{(\ell(k))}\right)\right), \lambda_{r(k)}^{\prime}=\lambda_{\max }^{\prime}\left(\mathscr{A}\left(\omega^{(r(k))}\right)\right)$, and solving this equation for $\omega^{(k+1)}$ yields

$$
\omega^{(k+1)}=\frac{\lambda_{\ell(k)}-\lambda_{r(k)}+\lambda_{r(k)}^{\prime} \omega^{(r(k))}-\lambda_{\ell(k)}^{\prime} \omega^{(\ell(k))}-\frac{\gamma}{2}\left(\left[\omega^{(r(k))}\right]^{2}-\left[\omega^{(\ell(k))}\right]^{2}\right)}{\left(\lambda_{r(k)}^{\prime}-\lambda_{\ell(k)}^{\prime}\right)-\gamma\left(\omega^{(r(k))}-\omega^{(\ell(k))}\right)} .
$$

By applications of Taylor's theorem with second order remainder to $\lambda_{r(k)}, \lambda_{\ell(k)}, \lambda_{r(k)}^{\prime}, \lambda_{\ell(k)}^{\prime}$, specifically by expressing them in terms of $\lambda_{\max }(\mathscr{A}(\omega))$ and one-sided derivatives of $\lambda_{\max }(\mathscr{A}(\omega))$ at $\omega_{*}$, we obtain

$$
\begin{gathered}
\omega^{(k+1)}=\left\{\left[\lambda_{*}+\lambda_{*,-}^{\prime}\left(\omega^{(\ell(k))}-\omega_{*}\right)+O\left(h^{2}\right)\right]-\left[\lambda_{*}+\lambda_{*,+}^{\prime}\left(\omega^{(r(k))}-\omega_{*}\right)+O\left(h^{2}\right)\right]+\right. \\
{\left[\lambda_{*,+}^{\prime}+\lambda_{*,+}^{\prime \prime}\left(\omega^{(r(k))}-\omega_{*}\right)+O\left(h^{2}\right)\right] \times\left[\left(\omega^{(r(k))}-\omega_{*}\right)+\omega_{*}\right]-} \\
\left.\left[\lambda_{*,-}^{\prime}+\lambda_{*,-}^{\prime \prime}\left(\omega^{(\ell(k))}-\omega_{*}\right)+O\left(h^{2}\right)\right] \times\left[\left(\omega^{(\ell(k))}-\omega_{*}\right)+\omega_{*}\right]-(\gamma / 2)\left[\left[\omega^{(r(k))}\right]^{2}-\left[\omega^{(\ell(k))}\right]^{2}\right]\right\} / \\
\left\{\left[\lambda_{*,+}^{\prime}+\lambda_{*,+}^{\prime \prime}\left(\omega^{(r(k))}-\omega_{*}\right)+O\left(h^{2}\right)\right]-\left[\lambda_{*,-}^{\prime}+\lambda_{*,-}^{\prime \prime}\left(\omega^{(\ell(k))}-\omega_{*}\right)+O\left(h^{2}\right)\right]-\gamma\left[\omega^{(r(k))}-\omega^{(\ell(k))}\right]\right\} .
\end{gathered}
$$

This can be rearranged into

$$
\omega^{(k+1)}=\frac{\left(\alpha_{+}-\alpha_{-}\right) \cdot \omega_{*}+\eta \cdot\left(\frac{\omega^{(\ell(k))}+\omega^{(r(k))}}{2}\right)}{\alpha_{+}-\alpha_{-}+\eta}+O\left(h^{2}\right)
$$

where

$$
\begin{aligned}
& \alpha_{+}:=\left[\lambda_{*,+}^{\prime}+\lambda_{*,+}^{\prime \prime}\left(\omega^{(r(k))}-\omega_{*}\right)\right], \\
& \alpha_{-}:=\left[\lambda_{*,-}^{\prime}+\lambda_{*,-}^{\prime \prime}\left(\omega^{(\ell(k))}-\omega_{*}\right)\right], \eta:=-\gamma\left(\omega^{(r(k))}-\omega^{(\ell(k))}\right) .
\end{aligned}
$$

The desired result follows from $\alpha_{+}-\alpha_{-}>0$, as indeed $\lambda_{*,+}^{\prime}-\lambda_{*,-}^{\prime}>0$ is a constant independent of $h$ so that

$$
\alpha_{+}-\alpha_{-}=\left(\lambda_{*,+}^{\prime}-\lambda_{*,-}^{\prime}\right)+O(h)=\Theta(1),
$$

and by setting $\alpha:=\left(\alpha_{+}-\alpha_{-}\right) /\left(\alpha_{+}-\alpha_{-}+\eta\right), \beta:=\eta /\left(\alpha_{+}-\alpha_{-}+\eta\right)$.

(iii) This is immediate from equation (3.5) by observing

$$
\begin{aligned}
\left|\omega^{(k+1)}-\omega_{*}\right| & =\left|\alpha \cdot \omega_{*}+\beta \cdot\left(\frac{\omega^{(\ell(k))}+\omega^{(r(k))}}{2}\right)-(\alpha+\beta) \cdot \omega_{*}\right|+O\left(h^{2}\right), \\
& =\left|\beta \cdot\left[\left(\frac{\omega^{(\ell(k))}+\omega^{(r(k))}}{2}\right)-\omega_{*}\right]\right|+O\left(h^{2}\right)=O\left(h^{2}\right) .
\end{aligned}
$$


The main rate-of-convergence result concerning Algorithm 1 in the non-smooth case is presented next. It asserts that if the maximum of the errors of the last two iterates is $h$, the error of the next iterate is $O\left(h^{2}\right)$. A similar rapid convergence conclusion is drawn for the sequence $\left\{\ell^{(k)}\right\}$ as well.

THEOREM 3.5 (Rate-of-convergence) Suppose that $\lambda_{\max }(\mathscr{A}(\omega)$ ) has a unique global minimizer over $\Omega$ at $\omega_{*}$, and that $\omega_{*} \in \operatorname{Int} \Omega$. Suppose also $\gamma<0$, the eigenvalue $\lambda_{\max }\left(\mathscr{A}\left(\omega_{*}\right)\right)$ is multiple, $0 \in$ Int $\partial \lambda_{\max }\left(\mathscr{A}\left(\omega_{*}\right)\right)$, and the sequence $\left\{\omega^{(k)}\right\}$ generated by Algorithm 1 is such that $\omega^{(k)} \neq \omega_{*}$ for all $k$. The following hold for $\left\{\omega^{(k)}\right\}$ for all $k$ large enough:

(i) $k \in\{\ell(k), r(k)\}$.

(ii) $\left|\omega^{(k+1)}-\omega_{*}\right|=O\left(\max \left\{\left|\omega^{(k)}-\omega_{*}\right|,\left|\omega^{(k-1)}-\omega_{*}\right|\right\}^{2}\right)$.

(iii) If $\gamma$ is large enough in absolute value, then $\lambda_{*}-\ell^{(k+1)}=O\left(\left(\lambda_{*}-\ell^{(k-1)}\right)^{2}\right)$.

Proof. (i) If $\omega^{(k)}>\omega_{*}$, then it is apparent from (3.5) that, for large $k$, we must have $\omega^{(k)} \in\left(\omega_{*},\left(\omega^{(\ell(k-1))}+\right.\right.$ $\left.\left.\omega^{(r(k-1))}\right) / 2\right)$ implying $\omega^{(k)}-\omega_{*}<\omega^{(r(k-1))}-\omega_{*}$. Hence, in this case, $r(k)=k$.

Similarly, if $\omega^{(k)}<\omega_{*}$, then $\left.\omega^{(k)} \in\left(\left(\omega^{(\ell(k-1))}+\omega^{(r(k-1))}\right) / 2\right), \omega_{*}\right)$ for large $k$ by (3.5). This in turn implies $\omega_{*}-\omega^{(k)}<\omega_{*}-\omega^{(\ell(k-1))}$, so $\ell(k)=k$.

(ii) Let us suppose $k=r(k)$ without loss of generality. (Otherwise, $k=\ell(k)$ by part (i) and a similar argument applies.) This means that $\ell(k) \neq k$, so $\ell(k)=\ell(k-1)$. If $\ell(k)=\ell(k-1)=k-1$, then part (iii) of Lemma 3.2 implies

$$
\left|\omega^{(k+1)}-\omega_{*}\right|=O\left(\max \left\{\omega_{*}-\omega^{(\ell(k))}, \omega^{(r(k))}-\omega_{*}\right\}^{2}\right)=O\left(\max \left\{\omega_{*}-\omega^{(k-1)}, \omega^{(k)}-\omega_{*}\right\}^{2}\right) .
$$

Hence, let us suppose $\ell(k-1) \neq k-1$. But then $r(k-1)=k-1$ by part (i). Furthermore, as $\omega^{(r(k))}=$ $\omega^{(k)} \in\left(\omega_{*},\left(\omega^{(\ell(k-1))}+\omega^{(r(k-1))}\right) / 2\right)$, we must have

$$
\omega^{(k-1)}-\omega_{*}=\omega^{(r(k-1))}-\omega_{*}>\omega_{*}-\omega^{(\ell(k-1))}=\omega_{*}-\omega^{(\ell(k))}
$$

from which we deduce

$$
\max \left\{\left|\omega^{(k-1)}-\omega_{*}\right|,\left|\omega^{(k)}-\omega_{*}\right|\right\} \geqslant \max \left\{\omega_{*}-\omega^{(\ell(k))}, \omega^{(r(k))}-\omega_{*}\right\} .
$$

Hence, letting $h:=\max \left\{\left|\omega^{(k-1)}-\omega_{*}\right|,\left|\omega^{(k)}-\omega_{*}\right|\right\}$, we have $\max \left\{\omega_{*}-\omega^{(\ell(k))}, \omega^{(r(k))}-\omega_{*}\right\}=O(h)$. It follows from part (iii) of Lemma 3.2 that $\left|\omega^{(k+1)}-\omega_{*}\right|=O\left(h^{2}\right)$, completing the proof.

(iii) Part (i) of Lemma 3.2 asserts that the point $\omega^{(k-1)}$ is the intersection point of $q_{\ell(k-2)}(\omega)$ and $q_{r(k-2)}(\omega)$. Without loss of generality, let us assume $\omega^{(r(k-2))}-\omega_{*}>\omega_{*}-\omega^{(\ell(k-2))}$. We have

$$
\begin{aligned}
\ell^{(k-1)} & =q_{r(k-2)}\left(\omega^{(k-1)}\right) \\
& =\lambda_{r(k-2)}+\lambda_{r(k-2)}^{\prime}\left(\omega^{(k-1)}-\omega^{r((k-2)}\right)+\frac{\gamma}{2}\left(\omega^{(k-1)}-\omega^{(r(k-2))}\right)^{2} .
\end{aligned}
$$

Now applications of Taylor's theorem about $\omega_{*}$ yield

$$
\begin{gathered}
\ell^{(k-1)}=\left[\lambda_{*}+\lambda_{*,+}^{\prime}\left(\omega^{(r(k-2))}-\omega_{*}\right)+\frac{\lambda_{*,+}^{\prime \prime}}{2}\left(\omega^{(r(k-2))}-\omega_{*}\right)^{2}\right]+ \\
{\left[\lambda_{*,+}^{\prime}+\lambda_{*,+}^{\prime \prime}\left(\omega^{(r(k-2))}-\omega_{*}\right)\right]\left(\omega^{(k-1)}-\omega^{(r(k-2))}\right)+\frac{\gamma}{2}\left(\omega^{(k-1)}-\omega^{(r(k-2))}\right)^{2}} \\
+O\left(\left(\omega^{(r(k-2))}-\omega_{*}\right)^{3}\right),
\end{gathered}
$$


where we use $\omega^{(k-1)}-\omega_{*}=O\left(\left(\omega^{(r(k-2))}-\omega_{*}\right)^{2}\right)$ due to part (iii) of Lemma 3.2. Letting $h:=\lambda_{*}-$ $\ell^{(k-1)}$, the last equation yields

$$
\begin{gathered}
h=-\lambda_{*,+}^{\prime}\left(\omega^{(k-1)}-\omega_{*}\right)-\frac{\lambda_{*,+}^{\prime \prime}}{2}\left(\omega^{(r(k-2))}-\omega_{*}\right)^{2} \\
-\lambda_{*,+}^{\prime \prime}\left(\omega^{(r(k-2))}-\omega_{*}\right)\left(\omega^{(k-1)}-\omega^{(r(k-2))}\right) \\
-\frac{\gamma}{2}\left(\omega^{(k-1)}-\omega^{(r(k-2))}\right)^{2}+O\left(\left(\omega^{(r(k-2))}-\omega_{*}\right)^{3}\right) \\
=-\lambda_{*,+}^{\prime}\left(\omega^{(k-1)}-\omega_{*}\right)+\frac{\lambda_{*,+}^{\prime \prime}}{2}\left(\omega^{(r(k-2))}-\omega_{*}\right)^{2} \\
-\frac{\gamma}{2}\left(\omega^{(r(k-2))}-\omega_{*}\right)^{2}+O\left(\left(\omega^{(r(k-2))}-\omega_{*}\right)^{3}\right) .
\end{gathered}
$$

Assuming $\gamma$ is large enough, the terms on the right-hand side of the last equality are $\Theta\left(\left(\omega^{(r(k-2))}-\right.\right.$ $\left.\left.\omega_{*}\right)^{2}\right)$. Hence, $\left(\omega^{(r(k-2))}-\omega_{*}\right)^{2}=\Theta(h)$, that is $\omega^{(k-1)}-\omega_{*}=O\left(\left(\omega^{(r(k-2))}-\omega_{*}\right)^{2}\right)=O(h)$.

Letting $h_{2}:=\lambda_{*}-\ell^{(k)} \leqslant \lambda_{*}-\ell^{(k-1)}$ (notice that $\left\{\ell^{(k)}\right\}$ is increasing bounded from above by $\lambda_{*}$ ), and following similar steps, we also deduce $\omega^{(k)}-\omega_{*}=O\left(h_{2}\right)=O(h)$. Now it follows from part (ii) that $\left|\omega^{(k+1)}-\omega_{*}\right|=O\left(h^{2}\right)$. The point $\omega^{(k+1)}$ is the intersection point of $q_{\ell(k)}(\omega)$ and $q_{r(k)}(\omega)$, where $\ell(k)=k$ or $r(k)=k$, so

$$
\ell^{(k+1)}=\bar{q}_{k}\left(\omega^{(k+1)}\right)=q_{k}\left(\omega^{(k+1)}\right)=\lambda_{k}+\lambda_{k}^{\prime}\left(\omega^{(k+1)}-\omega^{(k)}\right)+\frac{\gamma}{2}\left(\omega^{(k+1)}-\omega^{(k)}\right)^{2} .
$$

Assume for now $\omega^{(k)}>\omega_{*}$. Recalling $\omega^{(k)}-\omega_{*}=O(h)$, and once again applying Taylor's theorem to $\lambda_{k}, \lambda_{k}^{\prime}$ about $\omega_{*}$ gives rise to

$$
\begin{aligned}
\ell^{(k+1)}= & {\left[\lambda_{*}+\lambda_{*,+}^{\prime}\left(\omega^{(k)}-\omega_{*}\right)+O\left(\left(\omega^{(k)}-\omega_{*}\right)^{2}\right)\right]+} \\
& {\left[\lambda_{*,+}^{\prime}+O\left(\omega^{(k)}-\omega_{*}\right)\right]\left(\omega^{(k+1)}-\omega^{(k)}\right)+\frac{\gamma}{2}\left(\omega^{(k+1)}-\omega^{(k)}\right)^{2} } \\
= & \lambda_{*}+\lambda_{*,+}^{\prime}\left(\omega^{(k+1)}-\omega_{*}\right)+O\left(h^{2}\right)=\lambda_{*}+O\left(h^{2}\right),
\end{aligned}
$$

which in turn implies $\lambda_{*}-\ell^{(k+1)}=O\left(h^{2}\right)$ as desired. If $\omega^{(k)}<\omega_{*}$, all of the equalities above still hold but by applying Taylor's theorem on the left-hand side of $\omega_{*}$. This results in the same expressions except that occurrences of $\lambda_{*,+}^{\prime}$ are replaced by $\lambda_{*,-}^{\prime}$.

REMARK 3.2 The rate-of-convergence result assumes that the global minimizer $\omega_{*}$ belongs to Int $\Omega$. As for the eigenvalue optimization problem (1.3) associated with the inner numerical radius, given two distinct $\theta_{1}, \theta_{2} \in[0,2 \pi)$, we could evaluate $\lambda_{\max }\left(A \cos \theta_{j}+B \sin \theta_{j}\right)$ for $j=1,2$. Unless the largest eigenvalues are equal at these two distinct points, letting $\widetilde{\theta}$ be one of $\theta_{1}, \theta_{2}$, whichever of these points lead to a larger largest eigenvalue, we could perform the optimization over $\Omega:=[\widetilde{\theta}, \widetilde{\theta}+2 \pi]$. Then the global minimizer of $\lambda_{\max }(A \cos \theta+B \sin \theta)$ over $\theta \in \Omega$ belongs to Int $\Omega$ as required by the rate-ofconvergence analysis above.

\subsection{Numerical Experiments}

All numerical experiments in this paper have been carried out in MATLAB 9.2.0.556344 (R2017a). They all involve the computation of the inner numerical radius, that is the minimization of $\lambda_{\max }(A(\theta):=$ 
$A \cos \theta+B \sin \theta)$ over $\theta \in[0,2 \pi]$ for a given pair of Hermitian matrices $A, B \in \mathbb{C}^{n \times n}$. We employ eigopt (see Section 10 in Mengi et al. (2014)), a Matlab implementation of Algorithm 1, for these minimization problems. This implementation generates a sequence of lower bounds and a sequence of upper bounds for the minimal value of $\lambda_{\max }(\mathscr{A}(\theta))$, and terminate when the bounds differ by less than a prescribed tolerance, which we set equal to $10^{-15}$ unless otherwise specified. Additionally, a global lower bound $\gamma$ on the second derivatives of the largest eigenvalue function needs to be supplied to eigopt. We set $\gamma=-\|A\|_{2}-\|B\|_{2}$ in all our numerical experiments; this is a theoretically sound choice as discussed above.

We have proven above that there exist constants $c_{1}, c_{2}$ such that the sequences $\left\{\ell^{(k)}\right\}$ and $\left\{\omega^{(k)}\right\}$ by Algorithm 1 satisfy the following for all $k$ large enough:

$$
\lambda_{*}-\ell^{(k+1)} \leqslant c_{1}\left(\lambda_{*}-\ell^{(k-1)}\right)^{2} \quad \text { and } \quad\left|\omega^{(k+1)}-\omega_{*}\right| \leqslant c_{2} \max \left\{\left|\omega^{(k)}-\omega_{*}\right|,\left|\omega^{(k-1)}-\omega_{*}\right|\right\}^{2} .
$$

In the logarithmic scale and by letting $e_{k}:=\lambda_{*}-\ell^{(k)}$ and $\xi_{k}:=\left|\omega^{(k)}-\omega_{*}\right|$, these inequalities could equivalently be expressed as

$$
\ln \left(e_{k+1}\right) \leqslant 2 \ln \left(e_{k-1}\right)+\ln \left(c_{1}\right) \quad \text { and } \quad \ln \left(\xi_{k+1}\right) \leqslant 2 \ln \left(\max \left\{\xi_{k}, \xi_{k-1}\right\}\right)+\ln \left(c_{2}\right) .
$$

On the examples that exhibit non-smoothness (i.e., the examples for which the largest eigenvalue is multiple at the global minimizer), we check whether these inequalities hold in practice at the later stages of the algorithm when the errors are extremely small. Hence, on these examples, we run the algorithm to a very high precision by employing Advanpix Multiprecision Computing Toolbox ${ }^{1}$, a high precision toolbox for Matlab. For these non-smooth examples, the tolerance for termination supplied to eigopt is much smaller than $10^{-15}$; the precise tolerance values are specified below.

Distance to a Nearest Definite Pair. Consider the matrices $A=\operatorname{diag}(-3: 3) \in \mathbb{R}^{7 \times 7}$ (i.e., $A$ is the $7 \times 7$ diagonal matrix with $a_{j j}=-3+(j-1)$ for $\left.j=1, \ldots, 7\right)$ and $B \in \mathbb{R}^{7 \times 7}$ defined by $b_{i j}=1 /(i+j)$ except $b_{11}=b_{77}=-1$. This is an indefinite Hermitian pair example taken from Cheng \& Higham (1999). We run Algorithm 1 to compute the inner numerical radius $\zeta(A, B)$ and the distance $d_{\delta}(A, B)$ for $\delta=10^{-8}$. The computed distance by the algorithm is $d_{\delta}(A, B)=0.8118872239262$. The left column in Figure 1 illustrates the field of values of $A+\mathrm{i} B, A+\Delta A_{*}+\mathrm{i}\left(B+\Delta B_{*}\right)$ and $\widetilde{A}+\mathrm{i} \widetilde{B}=e^{-\mathrm{i} \varphi}\left(A+\Delta A_{*}+\mathrm{i}\left(B+\Delta B_{*}\right)\right)$, where $\Delta A_{*}, \Delta B_{*}$ are the minimal normed perturbations that solve (1.2) given by (2.4), and $\varphi=\theta_{*}+\pi / 2$ for $\theta_{*}$ as in (2.1). Note that $\widetilde{B}$ is positive definite with $\lambda_{\min }(\widetilde{B})=10^{-8}$.

Comparison of Algorithm 1 with the Level-Set Method. Next we compare the performances of the level-set based and support based algorithms to compute the inner numerical radius of $A_{n}+\mathrm{i} B_{n}$ for various $n$, where $A_{n}$ is the Fiedler matrix and $B_{n}$ is the Moler matrix of size $n \times n$. In Table 1 , we provide CPU times and the number of iterations required by each algorithm. The reason why the levelset approach requires more time is that it computes all eigenvalues of matrix pencils of size $2 n \times 2 n$, whereas the support based algorithm computes only the largest eigenvalues of $n \times n$ matrices.

The Case when $\lambda_{\max }\left(A\left(\theta_{*}\right)\right)$ is not Simple. The next example illustrates the order of convergence for Algorithm 1 when the largest eigenvalue is not simple at the global minimizer. Consider the tridiagonal

\footnotetext{
${ }^{1}$ Available at http://www.advanpix.com
} 

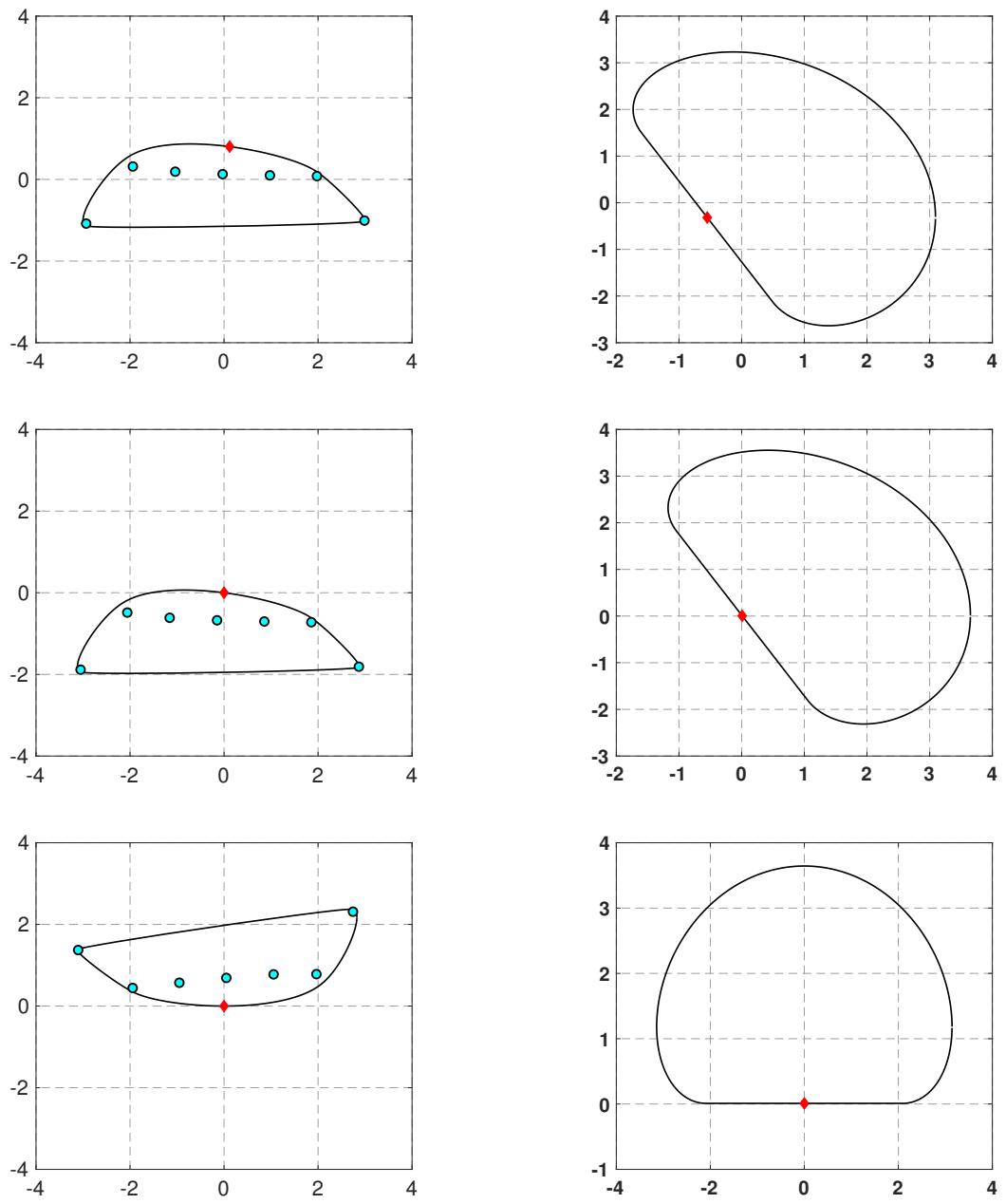

FIG. 1: The figure depicts the field of values of (Top) $A+\mathrm{i} B$, (Middle) $\left(A+\Delta A_{*}\right)+\mathrm{i}\left(B+\Delta B_{*}\right)$, (Bottom) $e^{-\mathrm{i} \varphi}\left(\left(A+\Delta A_{*}\right)+\mathrm{i}\left(B+\Delta B_{*}\right)\right)$, where $\Delta A_{*}, \Delta B_{*}, \varphi$ are as in (2.4), (2.1) for the given Hermitian pair $(A, B)$ and prescribed $\delta$. On the left column, $(A, B)$ is the Hermitian pair taken from Cheng \& Higham (1999) and $\delta=10^{-8}$, whereas, on the right column, $(A, B)$ is such that $A=\left(\widetilde{G}+\widetilde{G}^{*}\right) / 2$ and $B=-\mathrm{i}\left(\widetilde{G}-\widetilde{G}^{*}\right) / 2$ with $\widetilde{G}:=G e^{\mathrm{i} \pi / 6}, G$ denoting the $640 \times 640$ Grcar matrix, and $\delta=10^{-2}$. In each plot, the diamond marks the point where the inner numerical radius is attained, while, in the plots on the left, the circles mark the eigenvalues. 


\begin{tabular}{ccc||ccc}
\hline \hline \multicolumn{3}{c}{ LEVEL-SET } & \multicolumn{4}{c}{ SUPPORT-BASED } \\
$n$ & iter & $t$ & $n$ & iter & $t$ \\
\hline 120 & 5 & 1.60 & 120 & 84 & 0.40 \\
240 & 5 & 8.90 & 240 & 119 & 1.39 \\
360 & 6 & 45.44 & 360 & 143 & 4.04 \\
480 & 6 & 107.99 & 480 & 162 & 6.28 \\
\hline \hline
\end{tabular}

Table 1: CPU times (in seconds) and the number of iterations required by the level-set method and Algorithm 1 to compute the inner numerical radius of $A_{n}+\mathrm{i} B_{n}$ for various $n$, where $A_{n}$ is the Fiedler matrix and $B_{n}$ is the Moler matrix of size $n \times n$.

\begin{tabular}{c|c|c}
\hline \hline$k$ & $\ell^{(k+1)}$ & $\left|\omega^{(k+1)}-\theta_{*}\right|$ \\
\hline 12 & -1.000024850740653 & 0.000903071031368 \\
13 & -1.000002750999332 & 0.000041649882203 \\
14 & -1.000000001968154 & 0.000001828314169 \\
15 & -1.000000000011313 & 0.000000003906009 \\
16 & -1.000000000000000 & 0.000000000007542 \\
17 & -1.000000000000000 & 0.000000000000000 \\
\hline
\end{tabular}

Table 2: The sequence $\left\{\ell^{(k+1)}\right\}$ and the error of the sequence $\left\{\omega^{(k+1)}\right\}$ by Algorithm 1 are listed with respect to $k$ on the example involving the computation of the inner numerical radius of $C=\widetilde{C} e^{i \pi / 6}$, where $\widetilde{C}$ is as in (3.8).

matrix

$$
\widetilde{C}=\left[\begin{array}{ccccc}
1 & \mathrm{i} & & & \\
\mathrm{i} & 1 & \mathrm{i} & & \\
& \mathrm{i} & a_{3} & \ddots & \\
& & \ddots & \ddots & \mathrm{i} \\
& & & \mathrm{i} & a_{n}
\end{array}\right]+0.5 \mathrm{i} I_{n} \quad \text { with } a_{j}=2+\frac{j}{n}
$$

for $n=10$ and let $C=\widetilde{C} e^{\mathrm{i} \pi / 6}$. Letting $A:=\left(C+C^{*}\right) / 2, B:=-\mathrm{i}\left(C-C^{*}\right) / 2$, we have $C=A+\mathrm{i} B$. The global minimum of $\lambda_{\max }(A \cos \theta+B \sin \theta)$ is attained at $\theta_{*}=3.665191429188092$ and $\lambda_{\max }\left(A \cos \theta_{*}+\right.$ $B \sin \theta_{*}$ ) has multiplicity 2 . We observe a fast convergence for Algorithm 1 consistent with the rateof-convergence result in Theorem 3.5; Table 2 depicts this in double precision. We also perform the computations up until 600 decimal digit accuracy is satisfied, that is up until the lower and upper bounds by eigopt differ by an amount less than $10^{-600}$. On the left-column of Figure 2 the plot of $\ln \left(e_{2 k+1}\right)$ vs. $\ln \left(e_{2 k-1}\right)$, as well as $\ln \left(\xi_{k+1}\right)$ vs. $\ln \left(\max \left\{\xi_{k}, \xi_{k-1}\right\}\right)$ are provided. The plot of $\ln \left(e_{2 k+2}\right)$ vs. $\ln \left(e_{2 k}\right)$ turns out to be similar to the plot of $\ln \left(e_{2 k+1}\right)$ vs. $\ln \left(e_{2 k-1}\right)$, hence we omit it for brevity. The plots confirm our theoretical findings; in particular it is evident that the errors in the logarithmic scale decay linearly with slope two as expected in theory.

Linear Systems in Saddle Point Form. This is another example where the eigenvalue function is 
non-smooth at the optimizer. The matrix of a saddle point linear system is of the form

$$
\mathscr{A}=\left[\begin{array}{cc}
A & B^{T} \\
B & -C
\end{array}\right]
$$

where $A \in \mathbb{R}^{n \times n}$ is symmetric positive definite, $B \in \mathbb{R}^{m \times n}$ with $m \leqslant n$ and $C \in \mathbb{R}^{m \times m}$ is symmetric positive semidefinite. The matrix $\mathscr{A}$ is usually large and sparse, and can be reduced to the block diagonal matrix $\operatorname{diag}(A, S)$ with $S=-\left(C+B A^{-1} B^{T}\right)$ by row and column operations; indeed $X^{T} \mathscr{A} X=\operatorname{diag}(A, S)$ for some invertible $X \in \mathbb{R}^{(n+m) \times(n+m)}$. Now observe that $S$ is symmetric negative semidefinite implying that $\mathscr{A}$ is indefinite with $n$ positive eigenvalues and $\operatorname{rank}(S)$ negative eigenvalues.

The indefiniteness of $\mathscr{A}$ is a hurdle for iterative solvers such as Krylov subspace methods; it slows down the convergence. It has been shown in Liesen \& Parlett (2008) that, letting $\mathscr{J}:=\operatorname{diag}\left(I_{n},-I_{m}\right)$, if there is a real scalar $\mu$ such that $\mathscr{M}(\mu)=\mathscr{A}-\mu \mathscr{J}$ is positive definite or negative definite, then a conjugate gradient iteration that depends on this value of $\mu$ can be constructed to solve the linear system $\mathscr{A} x=b$. It is straightforward to verify that the positive definiteness or negative definiteness of $\mathscr{M}(\mu)$ for some $\mu \in \mathbb{R}$ is equivalent to the positive definiteness of $-\mathscr{A} \sin \theta+\mathscr{J} \cos \theta$ for some $\theta \in[0,2 \pi]$. Now the discussions in Section 2.1 suggests testing the definiteness of the pair $(\mathscr{A}, \mathscr{J})$. If this pair is definite, then, by those discussions, the matrix $-\mathscr{A} \sin \varphi+\mathscr{J} \cos \varphi$ is positive definite with $\lambda_{\min }(-\mathscr{A} \sin \varphi+\mathscr{J} \cos \varphi)=\gamma(\mathscr{A}, \mathscr{J})$ for $\varphi:=-\pi / 2+\phi$, where $\phi$ is the angle such that $\gamma(\mathscr{A}, \mathscr{J}) e^{\mathrm{i} \phi}$ is the point in $F(\mathscr{A}+\mathrm{i} \mathscr{J})$ closest to the origin. This in turn implies that $\mathscr{M}(\mu)$ is positive definite or negative definite for $\mu:=\cos \varphi / \sin \varphi$ depending on whether $\sin \varphi$ is negative or positive.

We consider a linear system in saddle point form that arises from a stable discretization of a Stokes equation with the coefficient matrix $\mathscr{A}$ of the form (3.9). This linear system is generated by the MATLAB package "Incompressible Flow Iterative Solution Software (IFISS) version 3.5" Elman et al. (2007); the sparse matrices $A, B, C$ are constructed by running the script file stokes_testproblem with the default options, resulting in $A$ of size $n=578$ and $C$ of size $m=256$. For compatibility with the particular examples worked through in Liesen \& Parlett (2008); Guo et al. (2009), we shift $A$ by $0.0764 I_{n}$, and run support based algorithm to determine the definiteness of the pair $(\mathscr{A}, \mathscr{J})$. We detect that the pair is definite in the light of the discussions at the end of Section 2.2, as it turns out $\min _{\theta \in[0,2 \pi]} \lambda_{\max }(\mathscr{A} \cos \theta+\mathscr{J} \sin \theta)<0$. Moreover computations yield $\mu=0.0541$ for which $\mathscr{M}(\mu)$ is positive definite, indeed $\lambda_{\min }(\mathscr{M}(\mu))=0.0222$. The non-smoothness in these computations is encountered in a strong fashion, as $\lambda_{\max }(\mathscr{A} \cos \theta+\mathscr{J} \sin \theta)$ at the minimizing $\theta$ has multiplicity three. Table 3 indicates rapid convergence for the sequences $\left\{\ell^{(k)}\right\}$ and $\left\{\omega^{(k)}\right\}$ by Algorithm 1. In particular, according to the table, the sequences $\left\{\ell^{(2 k)}\right\},\left\{\ell^{(2 k+1)}\right\}$ appear to be converging at a quadratic rate. Additionally, the number of accurate decimal digits of $\omega^{(k)}$ is doubled at every two iterations. We also see that these observations do hold in a higher precision on the right-hand column of Figure 2, where the plots are constructed when 400 decimal digit accuracy is required in the computed results. Once again, these are consistent with the assertions of Theorem 3.5. We omit the plot of $\ln \left(e_{2 k+2}\right)$ with respect to $\ln \left(e_{2 k}\right)$ in Figure 2 on the right-hand column because of its similarity to the plot illustrating $\ln \left(e_{2 k+1}\right)$ with respect to $\ln \left(e_{2 k-1}\right)$.

\section{Subspace Framework for Large-Scale Computation of Inner Numerical Radius}

We now deal with the general univariate eigenvalue optimization problems of the form (1.4) when the Hermitian matrices $A_{1}, \ldots, A_{\kappa}$ involved are of large size. This setting encompasses the eigenvalue optimization characterization (1.3) for the inner numerical radius when the matrices $A, B$ are large. 
Hence, the approach discussed in this section can be employed to determine the definiteness of a largescale Hermitian pair $(A, B)$, or for such a large definite Hermitian pair, it can be used to find an angle $\varphi$ such that $\widetilde{A}+\mathrm{i} \widetilde{B}=e^{-\mathrm{i} \varphi}(A+\mathrm{i} B)$ with $\lambda_{\min }(\widetilde{B})=\gamma(A, B)$.

Here we generalize the basic subspace procedure in Kangal et al. (2018); Kressner et al. (2018), taking into account also the non-smooth case when $\lambda_{\max }(\mathscr{A}(\omega))$ is multiple at the converged global minimizer. We prove that the convergence of the generalized subspace procedure occurs at a quadratic rate asymptotically in the non-smooth case. The previous works have established an R-order of convergence equal to $1+\sqrt{2}$ (see for instance Theorems 2.3 and 2.4 in Kressner et al. (2018)) in the smooth case, but left the rate-of-convergence issue in the non-smooth setting open.

\subsection{Generalized Subspace Procedure}

The subspace procedure operates on the reduced problems of the form

$$
\min _{\omega \in \Omega} \lambda_{\max }\left(\mathscr{A}^{\mathscr{V}}(\omega)\right),
$$

where

$$
\mathscr{A}^{\mathscr{V}}(\omega):=V^{*} \mathscr{A}(\omega) V=f_{1}(\omega) V^{*} A_{1} V+\cdots+f_{d}(\omega) V^{*} A_{d} V
$$

for a given small dimensional subspace $\mathscr{V}$, say $\operatorname{dim} \mathscr{V}=k$, and an $n \times k$ matrix $V$ whose columns form an orthonormal basis for $\mathscr{V}$. Note that (4.1) involves smaller $k \times k$ eigenvalue problems compared with (1.4), which involves $n \times n$ eigenvalue problems.

In the remaining part of this subsection, we shall describe a procedure to construct a small dimensional subspace $\mathscr{V}$ such that the global minimizers and the globally minimal values of $\lambda_{\max }(\mathscr{A}(\omega))$ and $\lambda_{\max }^{\mathscr{V}}(\mathscr{A}(\omega))$ are nearly the same. To this end, we remind a result from Sirkovic \& Kressner (2016); Kangal et al. (2018) that relates the eigenvalues of $\mathscr{A}(\omega)$ and $\mathscr{A}^{\mathscr{V}}(\omega)$. Here and throughout the rest of this section $\lambda_{j}(\mathscr{A}(\omega))$ and $v_{j}(\mathscr{A}(\omega))$ denote the $j$ th largest eigenvalue (counting the multiplicities) and a corresponding unit eigenvector of $\mathscr{A}(\omega)$.

LEMMA 4.1 Let $\mathscr{V}_{1}, \mathscr{V}_{2}$ be two subspaces of $\mathbb{C}^{n}$ such that $\mathscr{V}_{1} \subseteq \mathscr{V}_{2}$. We have the following:

(i) (Monotonicity) For each $k=1, \ldots, \operatorname{dim} \mathscr{V}_{1}$ and for all $\omega \in \Omega$, the inequalities $\lambda_{k}\left(\mathscr{A}^{\mathscr{V}_{1}}(\omega)\right) \leqslant$ $\lambda_{k}\left(\mathscr{A}^{\mathscr{1} / 2}(\omega)\right) \leqslant \lambda_{k}(\mathscr{A}(\omega))$ hold.

(ii) (Hermite Interpolation) For a given $\omega \in \Omega$, if $\mathscr{V}_{1}$ contains $v_{1}(\mathscr{A}(\omega)), \ldots, v_{j}(\mathscr{A}(\omega))$, then the following hold for each $k=1, \ldots, j$ :

- $\lambda_{k}\left(\mathscr{A}^{\mathscr{N} 1}(\omega)\right)=\lambda_{k}(\mathscr{A}(\omega))$.

- If $\lambda_{k}(\mathscr{A}(\omega))$ is simple, so is $\lambda_{k}\left(\mathscr{A}^{\mathscr{1}}(\omega)\right)$ and $\lambda_{k}^{\prime}\left(\mathscr{A}^{\mathscr{N} / 1}(\omega)\right)=\lambda_{k}^{\prime}(\mathscr{A}(\omega))$.

The subspace procedure is presented formally in Algorithm 2. At every iteration, the subspace procedure first solves a projected small-scale problem for a given subspace $\mathscr{V}$. Then, denoting the global minimizer of this small problem with $\widetilde{\omega}$, the subspace is expanded with the inclusion of an eigenvector corresponding to $\lambda_{\max }(\mathscr{A}(\widetilde{\omega}))$, as well as eigenvectors corresponding to other eigenvalues of $\mathscr{A}(\widetilde{\omega})$ that are at most $\varepsilon$ away from $\lambda_{\max }(\mathscr{A}(\widetilde{\omega}))$.

The procedures proposed in Kangal et al. (2018), Kressner et al. (2018) add only an eigenvector corresponding to the largest eigenvalue $\lambda_{\max }(\mathscr{A}(\widetilde{\omega}))$, but not the eigenvectors corresponding to nearby eigenvalues. If $\lambda_{\max }(\mathscr{A}(\widetilde{\omega}))$ is multiple or nearly multiple, practical implementations of Kangal et al. 


\begin{tabular}{c|c|c|}
\hline \hline$k$ & $\ell^{(k+1)}$ & $\left|\omega^{(k+1)}-\theta_{*}\right|$ \\
\hline 11 & -0.022231442152684 & 0.000008162291389 \\
12 & -0.022224901919276 & 0.000006757114932 \\
13 & -0.022224901723492 & 0.000000000136544 \\
14 & -0.022224901666671 & 0.000000000058712 \\
15 & -0.022224901666670 & 0.00000000000000 \\
\hline \hline
\end{tabular}

Table 3: This table is analogous to Table 2, but it concerns the example of the saddle point linear system arising from the Stokes equation, in particular the positive definiteness or negative definiteness of $\mathscr{A}-\mu \mathscr{J}$ for some $\mu$, where $\mathscr{A} \in \mathbb{R}^{(n+m) \times(n+m)}$ is as in (3.9) and $\mathscr{J}:=\operatorname{diag}\left(I_{n},-I_{m}\right)$.
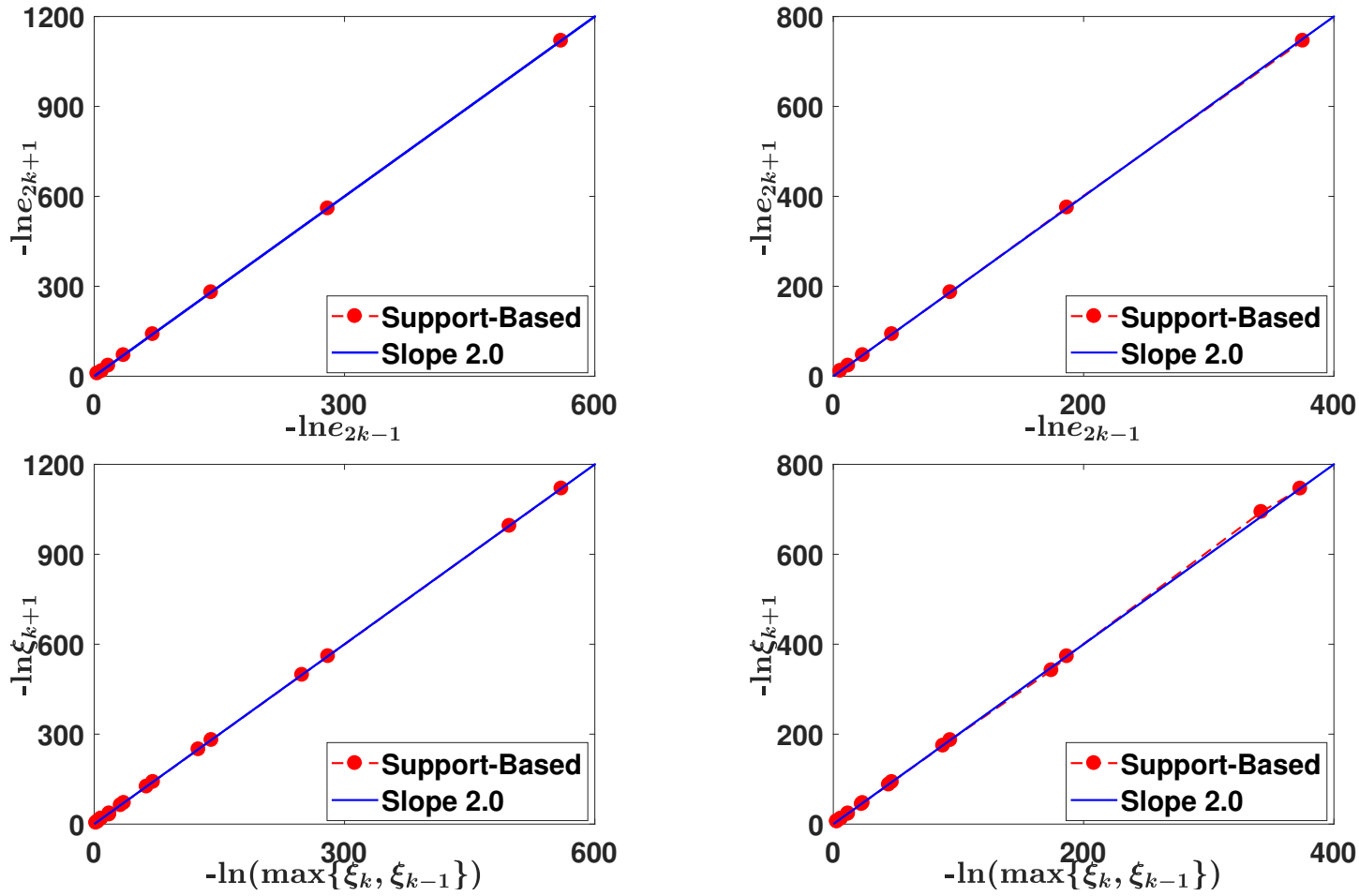

FIG. 2: The figure illustrates the convergence order of the support-based algorithm on the computation of the inner numerical radius (Left Column) of $C=\widetilde{C} e^{i \pi / 6}$ for $\widetilde{C}$ as in (3.8), and (Right Column) of the example related to the saddle point linear system arising from the Stokes equation. 


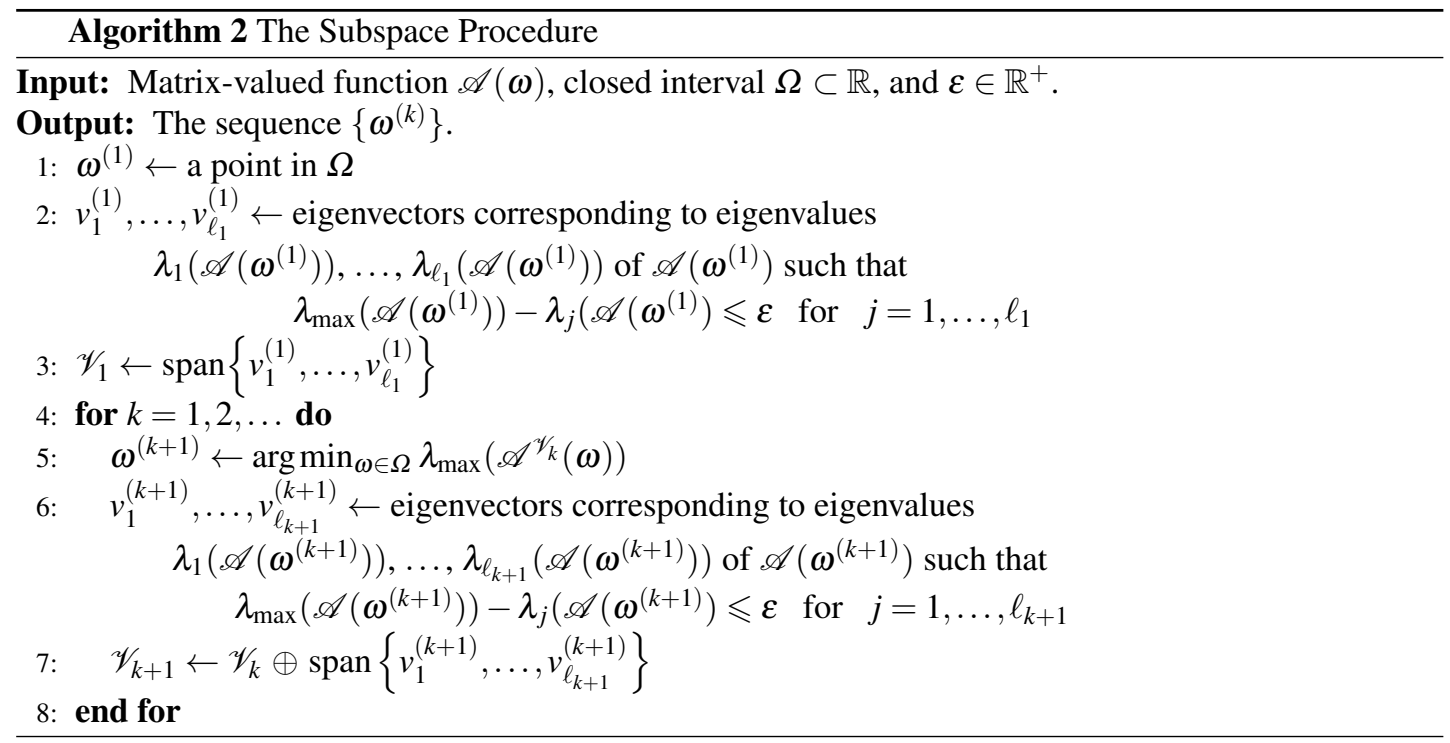

(2018), Kressner et al. (2018) resolve the ties based on rounding errors. This may result in eigenvectors added into the subspaces that are quite different, even if interpolation points (e.g., minimizers of the small-scale problems at two consecutive subspace iterations) are close. Algorithm 2 overcomes this issue by adding the whole subspace spanned by all of the eigenvectors corresponding to the eigenvalues close to the largest eigenvalues. This subspace appears to be better conditioned than the eigenvector corresponding to the largest eigenvalue in the case when $\lambda_{\max }(\mathscr{A}(\widetilde{\omega}))$ is multiple or nearly multiple.

The following interpolation result between the eigenvalues of the full and projected problems generated by Algorithm 2 is an immediate corollary of part (ii) of Lemma 4.1.

THEOREM 4.1 The following are satisfied by the sequences $\left\{\omega^{(k)}\right\}$ and $\left\{\mathscr{V}_{k}\right\}$ generated by Algorithm 2 for each $j$, each $k=1, \ldots, j$ and each $p=1, \ldots, \ell_{k}$ :

(i) $\lambda_{p}\left(\mathscr{A}^{\mathscr{V}}{ }_{j}\left(\omega^{(k)}\right)\right)=\lambda_{p}\left(\mathscr{A}\left(\omega^{(k)}\right)\right)$.

(ii) if $\lambda_{p}\left(\mathscr{A}\left(\omega^{(k)}\right)\right)$ is simple, then the same holds for $\lambda_{p}\left(\mathscr{A}^{\mathscr{V} j}\left(\omega^{(k)}\right)\right)$, and $\lambda_{p}^{\prime}\left(\mathscr{A}^{\mathscr{V}}{ }_{j}\left(\omega^{(k)}\right)\right)=\lambda_{p}^{\prime}\left(\mathscr{A}\left(\omega^{(k)}\right)\right)$.

In the case of the inner numerical radius, the projected small-scale problems can be solved globally and efficiently by means of the algorithms in Section 3. The support based algorithm in Section 3 is applicable to solve the projected problems associated with various other eigenvalue optimization problems of the form (1.4), as long as a global lower bound $\gamma$ on $\lambda_{\max }\left(\mathscr{A}^{\prime \prime}(\omega)\right)$ at the points of differentiability is available. The main computational burden of the subspace procedure stems from lines 2,6 , which require the computation of the eigenvectors of the full problem.

The subsequent two subsections are devoted to analyses of the convergence properties of Algorithm 2 . The next subsection provides formal arguments in support of the fact that every convergent subsequence of the sequence $\left\{\omega^{(k)}\right\}$ by the algorithm converges to a global minimizer of $\lambda_{\max }(\mathscr{A}(\omega))$. Then Section 4.3 addresses how quickly this convergence occurs. 


\subsection{Global Convergence}

Here and in the next subsection, we consider the problem at hand in the infinite dimensional setting. In particular, in this analysis the matrix-valued functions are replaced by self-adjoint compact operators $\mathscr{A}(\omega): \ell^{2}(\mathbb{N}) \rightarrow \ell^{2}(\mathbb{N})$, where $\ell^{2}(\mathbb{N})$ denotes the Hilbert space consisting of square summable infinite sequences of complex numbers equipped with the inner product $\langle v, w\rangle=\sum_{k=1}^{\infty} \bar{v}_{k} w_{k}$ and the norm $\|v\|=$ $\sqrt{\sum_{k=1}^{\infty}\left|v_{k}\right|^{2}}$. The compact self-adjoint operator $\mathscr{A}(\omega)$ dependent on the parameter $\omega$ is still assumed to be of the form specified in (1.4); only now $A_{j}: \ell^{2}(\mathbb{N}) \rightarrow \ell^{2}(\mathbb{N})$ are self-adjoint compact operators for $j=1, \ldots, d$. Intuitively $\mathscr{A}(\omega)$ for each $\omega$, as well as $A_{1}, \ldots, A_{\kappa}$, can be considered as infinite dimensional Hermitian matrices. This infinite dimensionality makes $\left\{\omega^{(k)}\right\}$ an infinite sequence so that, for instance, it is meaningful to analyze the order of convergence of this sequence as $k \rightarrow \infty$, as we do in the next subsection. In the finite dimensional case, when $\mathscr{A}(\omega)$ is a Hermitian matrix-valued function, the sequence $\left\{\omega^{(k)}\right\}$ is finite in exact arithmetic; however the rate-of-convergence analysis in the next subsection extends to the finite dimensional setting, but by letting $\omega^{(1)} \rightarrow \omega_{*}$ (for a global minimizer $\omega_{*}$ ) rather than $k \rightarrow \infty$. Such a practice is followed for instance in (Aliyev et al., 2017, Section 3) in the context of the large-scale computation of the $\mathscr{H}_{\infty}$-norm.

The global convergence of the subspace procedure is a consequence of the monotonicity and interpolation properties, as well as the uniform Lipschitz continuity of the reduced eigenvalue function formally stated below.

LEMMA 4.2 (Uniform Lipschitz Continuity, Kangal et al. (2018)) There exists a positive real number $\eta$ such that for a prescribed $J \in \mathbb{Z}^{+}$for each $j=1, \ldots, J$ and for all subspaces $\mathscr{V}$ of $\ell^{2}(\mathbb{N}$ ) (of dimension $J$ or greater) the following holds:

$$
\begin{gathered}
\left|\lambda_{j}\left(\mathscr{A}\left(\omega_{1}\right)\right)-\lambda_{j}\left(\mathscr{A}\left(\omega_{2}\right)\right)\right| \leqslant \eta\left|\omega_{1}-\omega_{2}\right| \quad \forall \omega_{1}, \omega_{2} \in \Omega \text {, and } \\
\left|\lambda_{j}\left(\mathscr{A}^{\mathscr{V}}\left(\omega_{1}\right)\right)-\lambda_{j}\left(\mathscr{A}^{\mathscr{V}}\left(\omega_{2}\right)\right)\right| \leqslant \eta\left|\omega_{1}-\omega_{2}\right| \quad \forall \omega_{1}, \omega_{2} \in \Omega,
\end{gathered}
$$

where $\lambda_{j}(\cdot)$ denotes the $j$ th largest eigenvalue of its Hermitian matrix argument.

Now let $\left\{\omega^{\left(\eta_{k}\right)}\right\}$ be a convergent subsequence of $\left\{\omega^{(k)}\right\}$. The interpolation property (part (i) of Theorem 4.1) implies

$$
\min _{\omega \in \Omega} \lambda_{\max }(\mathscr{A}(\omega)) \leqslant \lambda_{\max }\left(\mathscr{A}\left(\omega^{\left(\eta_{k}\right)}\right)\right)=\lambda_{\max }\left(\mathscr{A}^{\mathscr{V} \eta_{k}}\left(\omega^{\left(\eta_{k}\right)}\right)\right)
$$

while the monotonicity property (part (i) of Lemma 4.1) implies

$$
\begin{aligned}
\min _{\omega \in \Omega} \lambda_{\max }(\mathscr{A}(\omega)) & \geqslant \min _{\omega \in \Omega} \lambda_{\max }\left(\mathscr{A}^{\mathscr{V} \eta_{k+1}-1}(\omega)\right) \\
& =\lambda_{\max }\left(\mathscr{A}^{\mathscr{V} \eta_{k+1}-1}\left(\omega^{\left(\eta_{k+1}\right)}\right)\right) \geqslant \lambda_{\max }\left(\mathscr{A}^{\mathscr{V} \eta_{k}}\left(\omega^{\left(\eta_{k+1}\right)}\right)\right) .
\end{aligned}
$$

Thus, $\min _{\omega \in \Omega} \lambda_{\max }(\mathscr{A}(\omega))$ is squeezed between $\lambda_{\max }\left(\mathscr{A}^{\mathscr{V} \eta_{k}}\left(\omega^{\eta_{k}}\right)\right), \lambda_{\max }\left(\mathscr{A}^{\mathscr{V} \eta_{k}}\left(\omega^{\left(\eta_{k+1}\right)}\right)\right)$, the gap between which is decaying to zero as $k \rightarrow \infty$ due to uniform Lipschitz continuity (Lemma 4.2). This leads to the following global convergence result. The details of the proof are omitted, as the proof is identical to the one for Theorem 3.1 in Kangal et al. (2018).

THEOREM 4.2 (Global Convergence) Every convergent subsequence of the sequence $\left\{\omega^{(k)}\right\}$ generated by Algorithm 2 in the infinite dimensional setting converges to a global minimizer of $\lambda_{\max }(\mathscr{A}(\omega))$ over 
$\omega \in \Omega$. Moreover,

$$
\lim _{k \rightarrow \infty} \lambda_{\max }\left(\mathscr{A}^{\mathscr{Y} k}\left(\omega^{(k+1)}\right)\right)=\lim _{k \rightarrow \infty} \min _{\omega \in \Omega} \lambda_{\max }\left(\mathscr{A}^{\mathscr{N} k}(\omega)\right)=\min _{\omega \in \Omega} \lambda_{\max }(\mathscr{A}(\omega)) .
$$

\subsection{Rate-of-Convergence}

In this section, as has been done earlier for the rate-of-convergence analysis of the support based algorithm, we assume $\lambda_{\max }(\mathscr{A}(\omega))$ has a unique global minimizer over $\Omega$, say at $\omega_{*}$. Under this assumption, by employing Theorem 4.2, it follows that the sequence $\left\{\omega^{(k)}\right\}$ itself converges to the unique global minimizer $\omega_{*}=\arg \min _{\omega \in \Omega} \lambda_{\max }(\mathscr{A}(\omega))$.

Here we are concerned with how quickly $\left\{\omega^{(k)}\right\}$ by Algorithm 2 converges to $\omega_{*}$. If the eigenvalue $\lambda_{\max }\left(\mathscr{A}\left(\omega_{*}\right)\right)$ is simple, then the eigenvalue function $\lambda_{\max }(\mathscr{A}(\omega))$ is real analytic at $\omega_{*}$. In this case, it has been shown in Kressner et al. (2018) that the precise R-order of convergence of $\left\{\omega^{(k)}\right\}$ to $\omega_{*}$ is $1+\sqrt{2}$, i.e., there exists a sequence $\left\{\varepsilon^{(k)}\right\}$ converging to zero such that $\left|\omega^{(k)}-\omega_{*}\right| \leqslant \varepsilon^{(k)}$ for all $k$ large enough and $\varepsilon^{(k+1)}=O\left(\left(\varepsilon^{(k)}\right)^{1+\sqrt{2}}\right)$.

Otherwise, $\lambda_{\max }\left(\mathscr{A}\left(\omega_{*}\right)\right)$ is multiple with algebraic multiplicity $K \geqslant 2$. Throughout this section, it is assumed that $\omega_{*} \in \operatorname{Int} \Omega$, as well as

$$
0 \notin \operatorname{bd} \partial \lambda_{j}\left(\mathscr{A}\left(\omega_{*}\right)\right), \quad \text { for } j=1, \ldots, K,
$$

where $\lambda_{j}(\mathscr{A}(\omega))$ denotes the $j$ th largest eigenvalue of $\mathscr{A}(\omega)$, and bd $\partial \lambda_{j}(\mathscr{A}(\omega))$ denotes the boundary of the generalized Clarke derivative $\partial \lambda_{j}(\mathscr{A}(\omega))$ (we refer to (3.3) for the definition of the generalized Clarke derivative). This assumption is equivalent to having non-zero one-sided derivatives of $\lambda_{j}(\mathscr{A}(\omega))$ at $\omega_{*}$ for $j=1, \ldots, K$, and holds generically in the non-smooth case. As discussed in Section 3.2, $\lambda_{\max }(\mathscr{A}(\omega))$ is continuous, piece-wise real analytic at $\omega_{*}$, but usually not differentiable. Furthermore, part (i) of Lemma 3.1 asserts the existence of real analytic eigenvalue functions $\widetilde{\lambda}_{1}(\mathscr{A}(\omega)), \ldots, \widetilde{\lambda}_{n}(\mathscr{A}(\omega))$ of $\mathscr{A}(\omega)$. Precisely $K$ of these $n$ real analytic functions at $\omega_{*}$ must be equal to $\lambda_{\max }\left(\mathscr{A}\left(\omega_{*}\right)\right)$ with non-zero derivatives; a zero derivative for one of these $K$ functions at $\omega_{*}$ contradicts (4.5). Hence, without loss of generality, suppose

$$
\tilde{\lambda}_{1}\left(\mathscr{A}\left(\omega_{*}\right)\right)=\ldots=\tilde{\lambda}_{K}\left(\mathscr{A}\left(\omega_{*}\right)\right)=\lambda_{\max }\left(\mathscr{A}\left(\omega_{*}\right)\right)
$$

are such that

$$
\tilde{\lambda}_{1}^{\prime}\left(\mathscr{A}\left(\omega_{*}\right)\right), \ldots, \tilde{\lambda}_{P}^{\prime}\left(\mathscr{A}\left(\omega_{*}\right)\right)>0, \quad \tilde{\lambda}_{P+1}^{\prime}\left(\mathscr{A}\left(\omega_{*}\right)\right), \ldots, \tilde{\lambda}_{K}^{\prime}\left(\mathscr{A}\left(\omega_{*}\right)\right)<0 .
$$

Our arguments make use of the gap

$$
\begin{aligned}
\varphi & :=\lambda_{\max }\left(\mathscr{A}\left(\omega_{*}\right)\right)-\lambda_{K+1}\left(\mathscr{A}\left(\omega_{*}\right)\right) \\
& =\lambda_{\max }\left(\mathscr{A}\left(\omega_{*}\right)\right)-\max \left\{\tilde{\lambda}_{j}\left(\mathscr{A}\left(\omega_{*}\right)\right) \mid j=K+1, \ldots, n\right\},
\end{aligned}
$$

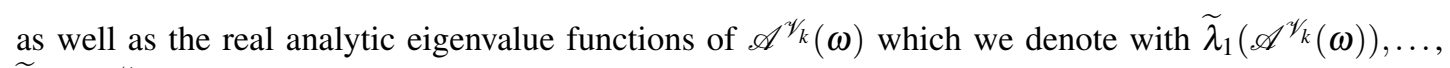
$\widetilde{\lambda}_{d_{k}}\left(\mathscr{A}^{\mathscr{V}}{ }^{k}(\omega)\right)$, where $d_{k}:=\operatorname{dim} \mathscr{V}_{k}$.

The Hermite interpolation property extends to $\widetilde{\lambda}_{j}(\mathscr{A}(\omega))$ and $\tilde{\lambda}_{j}\left(\mathscr{A}^{\mathscr{N} k}(\omega)\right)$ at the iterates $\omega^{(k)}$ of Algorithm 2 for large $k$ in the way stated by Lemma 4.3 below. This result immediately follows from part (ii) of Lemma 4.1, as the set $\left\{\widetilde{\lambda}_{j}(\mathscr{A}(\omega)) \mid j=1, \ldots, K\right\}$ corresponds to the set of largest $K$ eigenvalues of $\mathscr{A}(\omega)$ for all $\omega$ in an open interval $\mathscr{I}$ containing $\omega_{*}$. Furthermore, $\omega^{(k)} \in \mathscr{I}$ for large $k$, and the eigenvectors corresponding to $\widetilde{\lambda}_{j}\left(\mathscr{A}\left(\omega^{(k)}\right)\right)$ for $j=1, \ldots, K$ are included in the subspaces. 
LEMMA 4.3 Let $\left\{\omega^{(k)}\right\}$ be the sequence generated by Algorithm 2, and $\omega_{*}$ denote the unique global minimizer of $\lambda_{\max }(\mathscr{A}(\omega))$ over $\Omega$ such that $\lambda_{\max }\left(\mathscr{A}\left(\omega_{*}\right)\right)$ has algebraic multiplicity $K \geqslant 2$. For each $k \in \mathbb{Z}^{+}$large enough, there exist $\ell_{1}, \ldots, \ell_{K} \in\left\{1, \ldots, \operatorname{dim} \mathscr{V}_{k}\right\}$ satisfying

$$
\tilde{\lambda}_{j}\left(\mathscr{A}\left(\omega^{(k)}\right)\right)=\tilde{\lambda}_{\ell_{j}}\left(\mathscr{A}^{\mathscr{N} k}\left(\omega^{(k)}\right)\right), \quad \tilde{\lambda}_{j}^{\prime}\left(\mathscr{A}\left(\omega^{(k)}\right)\right)=\tilde{\lambda}_{\ell_{j}}^{\prime}\left(\mathscr{A}^{\mathscr{Y} k}\left(\omega^{(k)}\right)\right) \quad \text { for } j=1, \ldots, K .
$$

It is a matter of convention how we label the real analytic eigenvalue functions of $\mathscr{A}^{\mathscr{4} k}(\omega)$. For ease of notation, from here on, we relabel if necessary so that $\ell_{j}=j$ for $j=1, \ldots, K$ in Lemma 4.3. The next lemma gives a description of $\omega^{(k+1)}$, the global minimizer of $\lambda_{\max }\left(\mathscr{A}^{2 / k}(\omega)\right)$, in terms of the real analytic eigenvalues $\widetilde{\lambda}_{1}\left(\mathscr{A}^{\mathscr{T} k}(\omega)\right), \ldots, \tilde{\lambda}_{K}\left(\mathscr{A}^{\mathscr{T} k}(\omega)\right)$.

Lemma 4.4 Suppose that $\lambda_{\max }(\mathscr{A}(\omega))$ has a unique global minimizer over $\Omega$ at $\omega_{*}$, and that $\omega_{*} \in$ Int $\Omega$. Furthermore, suppose $\lambda_{\max }\left(\mathscr{A}\left(\omega_{*}\right)\right)$ has algebraic multiplicity $K \geqslant 2$, and that (4.5) holds. The following assertions are satisfied for all $k$ large enough:

(i) Letting $\delta_{j}:=\widetilde{\lambda}_{j}^{\prime}\left(\mathscr{A}\left(\omega_{*}\right)\right)>0$ for $j=1, \ldots, P$, there exists $\varepsilon_{j}>0$ such that for all $\omega \in\left(\omega_{*}-\right.$ $\left.\varepsilon_{j}, \omega_{*}+\varepsilon_{j}\right)$ we have

$$
\tilde{\lambda}_{j}^{\prime}(\mathscr{A}(\omega)) \geqslant 3 \delta_{j} / 4 \quad \text { and } \quad \tilde{\lambda}_{j}^{\prime}\left(\mathscr{A}^{\mathscr{1} k}(\omega)\right) \geqslant \delta_{j} / 2
$$

(ii) Letting $\delta_{j}:=\tilde{\lambda}_{j}^{\prime}\left(\mathscr{A}\left(\omega_{*}\right)\right)<0$ for $j=P+1, \ldots, K$, there exists $\varepsilon_{j}>0$ such that for all $\omega \in$ $\left(\omega_{*}-\varepsilon_{j}, \omega_{*}+\varepsilon_{j}\right)$ we have

$$
\tilde{\lambda}_{j}^{\prime}(\mathscr{A}(\omega)) \leqslant 3 \delta_{j} / 4 \quad \text { and } \quad \tilde{\lambda}_{j}^{\prime}\left(\mathscr{A}^{\mathscr{1} k}(\omega)\right) \leqslant \delta_{j} / 2
$$

(iii) The point $\omega^{(k+1)}$ is such that

$$
\lambda_{\max }\left(\mathscr{A}^{\mathscr{1} k}\left(\omega^{(k+1)}\right)\right)=\tilde{\lambda}_{j_{1}}\left(\mathscr{A}^{\mathscr{N} k}\left(\omega^{(k+1)}\right)\right)=\tilde{\lambda}_{j_{2}}\left(\mathscr{A}^{\mathscr{1} k}\left(\omega^{(k+1)}\right)\right)
$$

for some $j_{1} \in\{1, \ldots, P\}$ and some $j_{2} \in\{P+1, \ldots, K\}$.

Proof. (i) It follows from Proposition 2.9 in Kangal et al. (2018) that there exists an open interval $\mathscr{I} \subseteq$ Int $\Omega$ containing $\omega_{*}$ and a positive constant $v \in \mathbb{R}^{+}$such that $\widetilde{\lambda}_{j}^{\prime \prime}(\mathscr{A}(\omega))$, as well as $\widetilde{\lambda}_{j}^{\prime \prime}\left(\mathscr{A}^{\mathscr{V} k}(\omega)\right)$ for all $k$ sufficiently large are bounded in absolute value by $v$ uniformly over all $\omega \in \mathscr{I}$ and over all such large $k$.

The uniform boundedness of $\left|\widetilde{\lambda}_{j}^{\prime \prime}(\mathscr{A}(\omega))\right|$ over $\mathscr{I}$ combined with $\widetilde{\lambda}_{j}^{\prime}\left(\mathscr{A}\left(\omega_{*}\right)\right)=\delta_{j}$ imply the existence of $\widehat{\mathscr{I}}:=\left(\omega_{*}-\hat{\varepsilon}, \omega_{*}+\hat{\varepsilon}\right) \subseteq \mathscr{I}$ such that $\tilde{\lambda}_{j}^{\prime}(\mathscr{A}(\omega)) \geqslant 3 \delta_{j} / 4 \forall \omega \in \widehat{\mathscr{I}}$.

Since the global minimizer $\omega_{*}$ is assumed to be unique, by Lemma 3.4 we have $\omega^{(k)} \rightarrow \omega_{*}$ as $k \rightarrow \infty$. Hence, choose $k$ large enough so that $\omega^{(k)} \in \widehat{\mathscr{I}} / 2:=\left(\omega_{*}-\hat{\varepsilon} / 2, \omega_{*}+\hat{\varepsilon} / 2\right)$. Now we employ the Hermite interpolation property (Lemma 4.3) to deduce

$$
\tilde{\lambda}_{j}^{\prime}\left(\mathscr{A}^{\mathscr{1} k}\left(\omega^{(k)}\right)\right)=\tilde{\lambda}_{j}^{\prime}\left(\mathscr{A}\left(\omega^{(k)}\right)\right) \geqslant 3 \delta_{j} / 4
$$

for all such large $k$. Since $\left|\widetilde{\lambda}_{j}^{\prime \prime}\left(\mathscr{A}^{\mathscr{T} / k}(\omega)\right)\right|$ is also uniformly bounded in $\widehat{\mathscr{I}}$ by a constant independent of $k$, there exists $\widetilde{\mathscr{I}}:=\left(\omega^{(k)}-\widetilde{\mathcal{\varepsilon}}, \omega^{(k)}+\widetilde{\mathcal{\varepsilon}}\right) \subseteq \widehat{\mathscr{I}}$, in particular an $\widetilde{\mathcal{\varepsilon}} \in \mathbb{R}^{+}$independent of $k$, such that

$$
\tilde{\lambda}_{j}^{\prime}\left(\mathscr{A}^{\mathscr{Y} k}(\omega)\right) \geqslant \delta_{j} / 2 \quad \forall \omega \in \tilde{\mathscr{I}}
$$


for all such large $k$. By choosing $k$ even larger if necessary, it can be ensured that $\left|\omega^{(k)}-\omega_{*}\right|<\widetilde{\varepsilon} / 2$ so that $\left(\omega_{*}-\varepsilon_{j}, \omega_{*}+\varepsilon_{j}\right) \subseteq \widetilde{I}$ for $\varepsilon_{j}:=\widetilde{\varepsilon} / 2$.

(ii) This can be proven in a way similar to part (i).

(iii) Setting $\varepsilon:=\min \left\{\varepsilon_{j} \mid j=1, \ldots, K\right\}$ for $\varepsilon_{j}$ as in part (i) and (ii), there exists an open interval $\mathscr{I}=\left(\omega_{*}-\varepsilon, \omega_{*}+\varepsilon\right)$ such that for all large $k$ the following hold:

$$
\begin{array}{ll}
\tilde{\lambda}_{j}^{\prime}\left(\mathscr{A}^{\mathscr{1} k}(\omega)\right) \geqslant \delta_{j} / 2>0 \forall \omega \in \mathscr{I} & \text { for } j=1, \ldots, P, \\
\tilde{\lambda}_{j}^{\prime}\left(\mathscr{A}^{\mathscr{N} k}(\omega)\right) \leqslant \delta_{j} / 2<0 \forall \omega \in \mathscr{I} & \text { for } j=P+1, \ldots, K .
\end{array}
$$

Furthermore, let $\widetilde{\mathscr{I}}:=\left(\omega_{*}-\widetilde{\varepsilon}, \omega_{*}+\widetilde{\varepsilon}\right)$ for $\widetilde{\varepsilon}:=\min \{\varepsilon, \varphi /(8 \eta)\}$, where $\eta$ is the uniform Lipschitz constant in Lemma 4.2, and $\varphi$ is as in (4.6). Without loss of generality, we make the following two assumptions. First, the set $\left\{\widetilde{\lambda}_{1}(\mathscr{A}(\omega)), \ldots, \widetilde{\lambda}_{K}(\mathscr{A}(\omega))\right\}$ corresponds to the set of $K$ largest eigenvalues of $\mathscr{A}(\omega)$ for all $\omega \in \widetilde{\mathscr{I}}$. Secondly, $\omega^{(k)}, \omega^{(k+1)}:=\arg \min _{\omega \in \Omega} \lambda_{\max }\left(\mathscr{A}^{\mathscr{T} k}(\omega)\right) \in \widetilde{\mathscr{I}}$, since $\omega^{(k)} \rightarrow \omega_{*}$ as $k \rightarrow \infty$.

We start by showing that $\lambda_{\max }\left(\mathscr{A}^{\mathscr{Y} k}(\omega)\right)>\widetilde{\lambda}_{j^{\prime}}\left(\mathscr{A}^{\mathscr{1} k}(\omega)\right)$ for all $\omega \in \widetilde{\mathscr{I}}$ and for $j^{\prime}=K+1, \ldots, \operatorname{dim} \mathscr{V}_{k}$. To this end, first observe that

$$
\lambda_{K}(\mathscr{A}(\omega))-\lambda_{K+1}(\mathscr{A}(\omega)) \geqslant \lambda_{K}\left(\mathscr{A}\left(\omega_{*}\right)\right)-\lambda_{K+1}\left(\mathscr{A}\left(\omega_{*}\right)\right)-\varphi / 4=3 \varphi / 4
$$

for all $\omega \in \widetilde{\mathscr{I}}$, where we employ the Lipschitz continuity of $\lambda_{K}(\mathscr{A}(\omega))$ and $\lambda_{K+1}(\mathscr{A}(\omega))$ with the Lipschitz constant $\eta$ (see Lemma 4.2). In particular, for each $j \in\{1, \ldots, K\}$, we have

$$
3 \varphi / 4 \leqslant \tilde{\lambda}_{j}\left(\mathscr{A}\left(\omega^{(k)}\right)\right)-\lambda_{K+1}\left(\mathscr{A}\left(\omega^{(k)}\right)\right) \leqslant \tilde{\lambda}_{j}\left(\mathscr{A}^{\mathscr{1} / k}\left(\omega^{(k)}\right)\right)-\lambda_{K+1}\left(\mathscr{A}^{\mathscr{1} k}\left(\omega^{(k)}\right)\right)
$$

where in the second inequality we exploit the interpolation property (Lemma 4.3), and the monotonicity (i.e., $\lambda_{K+1}\left(\mathscr{A}\left(\omega^{(k)}\right)\right) \geqslant \lambda_{K+1}\left(\mathscr{A}^{\mathscr{N} / k}\left(\omega^{(k)}\right)\right)$ due to part (i) of Lemma 4.1). Now the last inequality implies

$$
\tilde{\lambda}_{j}\left(\mathscr{A}^{\mathscr{1} k}(\omega)\right)-\lambda_{K+1}\left(\mathscr{A}^{\mathscr{1} / k}(\omega)\right) \geqslant \tilde{\lambda}_{j}\left(\mathscr{A}^{\mathscr{1} k}\left(\omega^{(k)}\right)\right)-\lambda_{K+1}\left(\mathscr{A}^{\mathscr{T} k}\left(\omega^{(k)}\right)\right)-\varphi / 2=\varphi / 4
$$

for all $\omega \in \widetilde{\mathscr{I}}$. Note that above we make use of the uniform Lipschitz continuity of $\tilde{\lambda}_{j}\left(\mathscr{A}^{1 / k}(\omega)\right)$ and $\lambda_{K+1}\left(\mathscr{A}^{\mathscr{N}} k(\omega)\right)$ with the uniform Lipschitz constant $\eta$ independent of the subspace $\mathscr{V}_{k}$; the latter is immediate from Lemma 4.2, whereas the former can be seen from $\left|\widetilde{\lambda}_{j}^{\prime}\left(\mathscr{A}^{1 / k}(\omega)\right)\right| \leqslant\left\|\mathscr{A}^{\prime}(\omega)\right\|_{2}$. (Strictly speaking $\eta$ as in Lemma 4.2 is the uniform Lipschitz constant for the sorted eigenvalue $\lambda_{j}\left(\mathscr{A}^{\mathscr{2} k}(\omega)\right)$, but without loss of generality it can be chosen even larger if necessary so that it is also at least as large as the uniform Lipschitz constant for $\widetilde{\lambda}_{j}^{\prime}\left(\mathscr{A}^{\mathscr{N} k}(\omega)\right)$.) Inequality (4.7) means that $\lambda_{K+1}\left(\mathscr{A}^{\mathscr{N} k}(\omega)\right)$ is the largest of $\widetilde{\lambda}_{j^{\prime}}\left(\mathscr{A}^{\mathscr{Y}} k(\omega)\right)$ for $j^{\prime}=K+1, \ldots, \operatorname{dim} \mathscr{V}_{k}$ for $\omega \in \widetilde{\mathscr{I}}$. Consequently, for every $j^{\prime}=K+1, \ldots, \operatorname{dim} \mathscr{V}_{k}$ and for all $\omega \in \tilde{\mathscr{I}}$, we deduce

$$
\lambda_{\max }\left(\mathscr{A}^{\mathscr{1} k}(\omega)\right)-\tilde{\lambda}_{j^{\prime}}\left(\mathscr{A}^{\mathscr{Y} k}(\omega)\right) \geqslant \lambda_{\max }\left(\mathscr{A}^{\mathscr{N} k}(\omega)\right)-\lambda_{K+1}\left(\mathscr{A}^{\mathscr{Y} k}(\omega)\right) \geqslant \varphi / 4 .
$$

It follows from the previous paragraph that for all $\omega \in \tilde{\mathscr{I}}$ the following holds: $\lambda_{\max }\left(\mathscr{A}^{\mathscr{1} / k}(\omega)\right)=$ $\tilde{\lambda}_{j}\left(\mathscr{A}^{\mathscr{N} k}(\omega)\right)$ for some $j \in\{1, \ldots, K\}$, yet $\lambda_{\max }\left(\mathscr{A}^{\mathscr{T} k}(\omega)\right)>\widetilde{\lambda}_{j}\left(\mathscr{A}^{\mathscr{T} k}(\omega)\right)$ for $j \notin\{1, \ldots, K\}$. Now consider a point $\widetilde{\omega} \in \widetilde{I}$ such that

- $\lambda_{\max }\left(\mathscr{A}^{2 / k}(\widetilde{\boldsymbol{\omega}})\right)=\widetilde{\lambda}_{p_{1}}\left(\mathscr{A}^{2 / k}(\widetilde{\boldsymbol{\omega}})\right)=\cdots=\widetilde{\lambda}_{p_{q}}\left(\mathscr{A}^{2 / k}(\widetilde{\boldsymbol{\omega}})\right) \exists p_{1}, \ldots, p_{q} \in\{1, \ldots, P\}$ for some $q \geqslant 1$, yet $\lambda_{\max }\left(\mathscr{A}^{\mathscr{N} k}(\widetilde{\omega})\right)>\widetilde{\lambda}_{j}\left(\mathscr{A}^{\mathscr{N} k}(\widetilde{\omega})\right)$ for $j \notin\left\{p_{1}, \ldots, p_{q}\right\}$, or 
- $\lambda_{\max }\left(\mathscr{A}^{\mathscr{Y} k}(\widetilde{\omega})\right)=\widetilde{\lambda}_{n_{1}}\left(\mathscr{A}^{\mathscr{Y} k}(\widetilde{\omega})\right)=\cdots=\widetilde{\lambda}_{n_{s}}\left(\mathscr{A}^{\mathscr{T} k}(\widetilde{\omega})\right) \exists n_{1}, \ldots, n_{s} \in\{P+1, \ldots, K\}$ for some $s \geqslant 1$, yet $\lambda_{\max }\left(\mathscr{A}^{\mathscr{N} k}(\widetilde{\omega})\right)>\widetilde{\lambda}_{j}\left(\mathscr{A}^{\mathscr{N} k}(\widetilde{\omega})\right)$ for $j \notin\left\{n_{1}, \ldots, n_{s}\right\}$.

We shall show that such a point cannot be a minimizer of $\lambda_{\max }\left(\mathscr{A}^{4 / k}(\omega)\right)$. For these two cases, we respectively have

$$
\begin{aligned}
& \partial \lambda_{\max }\left(\mathscr{A}^{\mathscr{V} k}(\widetilde{\omega})\right)=\operatorname{Co}\left\{\widetilde{\lambda}_{p_{j}}^{\prime}\left(\mathscr{A}^{\mathscr{V} k}(\widetilde{\omega})\right) \mid j=1, \ldots, q\right\} \subseteq\left(\delta_{p, \min } / 2, \infty\right), \\
& \partial \lambda_{\max }\left(\mathscr{A}^{\mathscr{V} k}(\widetilde{\boldsymbol{\omega}})\right)=\operatorname{Co}\left\{\tilde{\lambda}_{n_{j}}^{\prime}\left(\mathscr{A}^{\mathscr{V} k}(\widetilde{\omega})\right) \mid j=1, \ldots, s\right\} \subseteq\left(-\infty, \delta_{n, \text { max}} / 2\right),
\end{aligned}
$$

where $\delta_{p, \text { min }}:=\min \left\{\delta_{p_{j}} \mid j=1, \ldots, q\right\}>0, \delta_{n, \max }:=\max \left\{\delta_{n_{j}} \mid j=1, \ldots, s\right\}<0$, and $\operatorname{Co}(S)$ denotes the convex hull of a set $S$. In either case, $0 \notin \partial \lambda_{\max }\left(\mathscr{A}^{\mathscr{T} / k}(\widetilde{\omega})\right)$ implying $\widetilde{\omega}$ is not a minimizer of $\lambda_{\max }\left(\mathscr{A}^{\mathscr{1} k}(\omega)\right)$. This means that, since $\omega^{(k+1)} \in \widetilde{\mathscr{I}}$ is a minimizer of $\lambda_{\max }\left(\mathscr{A}^{1 / k}(\omega)\right)$, we must have $\lambda_{\max }\left(\mathscr{A}^{\mathscr{1} k}\left(\omega^{(k+1)}\right)\right)=\widetilde{\lambda}_{j_{1}}\left(\mathscr{A}^{\mathscr{1} k}\left(\omega^{(k+1)}\right)\right)=\widetilde{\lambda}_{j_{2}}\left(\mathscr{A}^{\mathscr{1} k}\left(\omega^{(k+1)}\right)\right)$ for some $j_{1} \in\{1, \ldots, P\}$ and $j_{2} \in\{P+1, \ldots, K\}$.

Now we are ready to present the main quadratic rate-of-convergence result in the non-smooth setting; this result follows from the Hermite interpolation properties in Lemma 4.3, as well as Lemma 4.4.

Theorem 4.3 (Quadratic Convergence in the Non-Smooth Case) Suppose that the global minimizer $\omega_{*}:=\arg \min _{\omega \in \Omega} \lambda_{\max }(\mathscr{A}(\omega))$ is unique and such that $\omega_{*} \in \operatorname{Int} \Omega$, the eigenvalue $\lambda_{\max }\left(\mathscr{A}\left(\omega_{*}\right)\right)$ is multiple, condition (4.5) holds. The sequence $\left\{\omega^{(k)}\right\}$ generated by Algorithm 2 satisfies

$$
\left|\omega^{(k+1)}-\omega_{*}\right|=O\left(\left(\omega^{(k)}-\omega_{*}\right)^{2}\right)
$$

for all large $k$.

Proof. Part (i) and (ii) of Lemma 4.4 show the existence of an open interval $\mathscr{I}:=\left(\omega_{*}-\varepsilon, \omega_{*}+\varepsilon\right) \subseteq$ Int $\Omega$ where $\widetilde{\lambda}_{j}^{\prime}(\mathscr{A}(\omega))$ for $j=1, \ldots, P$ is bounded from below uniformly by a positive real number and $\tilde{\lambda}_{j}^{\prime}(\mathscr{A}(\omega))$ for $j=P+1, \ldots, K$ is bounded from above uniformly by a negative real number. Let us consider $k$ large enough so that $\omega^{(k)} \in \mathscr{I}$.

By part (iii) of Lemma 4.4 , there exist $j_{1} \in\{1, \ldots, P\}, j_{2} \in\{P+1, \ldots, K\}$ such that $\tilde{\lambda}_{j_{1}}\left(\mathscr{A}^{\mathscr{N} k}\left(\omega^{(k+1)}\right)\right)$ $=\widetilde{\lambda}_{j_{2}}\left(\mathscr{A}^{1 / k}\left(\omega^{(k+1)}\right)\right)$. For such a pair of $j_{1}, j_{2}$ define the real analytic functions

$$
\lambda(\mathscr{A}(\omega)):=\tilde{\lambda}_{j_{1}}(\mathscr{A}(\omega))-\tilde{\lambda}_{j_{2}}(\mathscr{A}(\omega)), \quad \lambda\left(\mathscr{A}^{\mathscr{T} k}(\omega)\right):=\tilde{\lambda}_{j_{1}}\left(\mathscr{A}^{\mathscr{1} k}(\omega)\right)-\tilde{\lambda}_{j_{2}}\left(\mathscr{A}^{\mathscr{T} k}(\omega)\right) .
$$

Observe that there exists a constant $\zeta \in \mathbb{R}^{+}$such that

$$
\lambda^{\prime}\left(\mathscr{A}\left(\omega^{(k)}\right)\right)=\tilde{\lambda}_{j_{1}}^{\prime}\left(\mathscr{A}\left(\omega^{(k)}\right)\right)-\tilde{\lambda}_{j_{2}}^{\prime}\left(\mathscr{A}\left(\omega^{(k)}\right)\right) \geqslant \zeta \quad \Longrightarrow\left|\left[\lambda^{\prime}\left(\mathscr{A}\left(\omega^{(k)}\right)\right)\right]^{-1}\right| \leqslant \zeta^{-1}
$$

independent of $k$. Note also that the real analyticity of $\widetilde{\lambda}_{j_{1}}(\mathscr{A}(\omega)), \widetilde{\lambda}_{j_{2}}(\mathscr{A}(\omega))$ implies the Lipschitz continuity of $\lambda^{\prime}(\mathscr{A}(\omega))$ on $\mathscr{I}$.

The proof manipulates the following equation:

$$
0=\lambda\left(\mathscr{A}\left(\omega_{*}\right)\right)=\lambda\left(\mathscr{A}\left(\omega^{(k)}\right)\right)+\int_{0}^{1} \lambda^{\prime}\left(\mathscr{A}\left(\omega^{(k)}+t\left(\omega_{*}-\omega^{(k)}\right)\right)\right)\left(\omega_{*}-\omega^{(k)}\right) d t .
$$


We employ $\lambda\left(\mathscr{A}\left(\omega^{(k)}\right)\right)=\lambda\left(\mathscr{A}^{\mathscr{V} k}\left(\omega^{(k)}\right)\right)$ (due to Lemma 4.3) in this equation, then multiply both sides by $\left[\lambda^{\prime}\left(\mathscr{A}\left(\omega^{(k)}\right)\right)\right]^{-1}$ to obtain

$$
\begin{array}{r}
0=\left[\lambda^{\prime}\left(\mathscr{A}\left(\omega^{(k)}\right)\right)\right]^{-1} \lambda\left(\mathscr{A}^{\mathscr{V} k}\left(\omega^{(k)}\right)\right)+\left(\omega_{*}-\omega^{(k)}\right)+\left[\lambda^{\prime}\left(\mathscr{A}\left(\omega^{(k)}\right)\right)\right]^{-1} \times \\
\int_{0}^{1}\left[\lambda^{\prime}\left(\mathscr{A}\left(\omega^{(k)}+t\left(\omega_{*}-\omega^{(k)}\right)\right)\right)-\lambda^{\prime}\left(\mathscr{A}\left(\omega^{(k)}\right)\right)\right]\left(\omega_{*}-\omega^{(k)}\right) d t .
\end{array}
$$

An application of Taylor's theorem to $\lambda\left(\mathscr{A}^{\mathscr{1} / k}(\omega)\right)$ about $\omega^{(k)}$ with second order remainder combined with the equalities $\lambda\left(\mathscr{A}^{1 / k}\left(\omega^{(k+1)}\right)\right)=0$ and $\lambda^{\prime}\left(\mathscr{A}^{1 / k}\left(\omega^{(k)}\right)\right)=\lambda^{\prime}\left(\mathscr{A}\left(\omega^{(k)}\right)\right)$ (a corollary of Lemma 4.3) lead us to

$$
\left[\lambda^{\prime}\left(\mathscr{A}\left(\omega^{(k)}\right)\right)\right]^{-1} \lambda\left(\mathscr{A}^{\mathscr{4} k}\left(\omega^{(k)}\right)\right)=\left(\omega^{(k)}-\omega^{(k+1)}\right)+O\left(\left(\omega^{(k)}-\omega^{(k+1)}\right)^{2}\right) .
$$

Now using the last equality in (4.9) gives rise to

$$
\begin{aligned}
0= & \left(\omega_{*}-\omega^{(k+1)}\right)+O\left(\left(\omega^{(k)}-\omega^{(k+1)}\right)^{2}\right)+\left[\lambda^{\prime}\left(\mathscr{A}\left(\omega^{(k)}\right)\right)\right]^{-1} \times \\
& \int_{0}^{1}\left[\lambda^{\prime}\left(\mathscr{A}\left(\omega^{(k)}+t\left(\omega_{*}-\omega^{(k)}\right)\right)\right)-\lambda^{\prime}\left(\mathscr{A}\left(\omega^{(k)}\right)\right)\right]\left(\omega_{*}-\omega^{(k)}\right) d t
\end{aligned}
$$

implying

$$
\begin{aligned}
& \left|\omega^{(k+1)}-\omega_{*}\right| \leqslant O\left(\left(\omega^{(k)}-\omega^{(k+1)}\right)^{2}\right)+\left|\left[\lambda^{\prime}\left(\mathscr{A}\left(\omega^{(k)}\right)\right)\right]^{-1}\right| \times \\
& \int_{0}^{1}\left|\lambda^{\prime}\left(\mathscr{A}\left(\omega^{(k)}+t\left(\omega_{*}-\omega^{(k)}\right)\right)\right)-\lambda^{\prime}\left(\mathscr{A}\left(\omega^{(k)}\right)\right)\right|\left|\omega_{*}-\omega^{(k)}\right| d t .
\end{aligned}
$$

Finally the desired equality (4.8) follows from (4.10) by employing $\left|\left[\lambda^{\prime}\left(\mathscr{A}\left(\omega^{(k)}\right)\right)\right]^{-1}\right| \leqslant \zeta^{-1}$, the Lipschitz continuity of $\lambda^{\prime}(\mathscr{A}(\omega))$, as well as the inequality

$$
\left(\omega^{(k)}-\omega^{(k+1)}\right)^{2} \leqslant 2\left[\left(\omega^{(k+1)}-\omega_{*}\right)^{2}+\left(\omega^{(k)}-\omega_{*}\right)^{2}\right] .
$$

REMARK 4.1 The eigenvalue function in (1.3) associated with the inner numerical radius, as well as its reduced counter-part, are periodic functions with periods $2 \pi$. Even if $\omega_{*}$ turns out to be an end-point of $\Omega=[0,2 \pi]$, the analysis and quadratic convergence result above apply to a sequence $\left\{\widetilde{\omega}^{(k)}\right\}$ such that $\omega^{(k)}=\widetilde{\omega}^{(k)} \bmod 2 \pi$ (the actual sequence generated $\left\{\omega^{(k)}\right\}$ is congruent to a sequence $\left\{\widetilde{\omega}^{(k)}\right\}$ generated by the algorithm when it is applied on an interval containing $\omega_{*}$ strictly in its interior). More generally, the assumption $\omega_{*} \in$ Int $\Omega$ is not essential for the quadratic convergence result above whenever the eigenvalue function has periodic nature.

\section{$4.4 \quad$ Numerical Experiments}

In this section we test Algorithm 2 on various examples involving the computation of the inner numerical radius (i.e., $\mathscr{A}(\omega)=A \cos \omega+B \sin \omega$ in all of the examples for a given pair $A, B \in \mathbb{C}^{n \times n}$ of Hermitian 
matrices), majority of which exhibit non-smoothness at the global minimizer. In all of these examples, the subspace framework is terminated when the condition $\left|\lambda_{\max }\left(\mathscr{A}^{\mathscr{T} / k}\left(\omega^{k+1}\right)\right)-\lambda_{\max }\left(\mathscr{A}^{\mathscr{V} k-1}\left(\omega^{k}\right)\right)\right|<$ tol is satisfied, where tol is a prescribed tolerance; we always set tol $=10^{-12}$ unless otherwise specified. Additionally, the input parameter $\varepsilon$ to Algorithm 2 is set equal to $10^{-16}\|\mathscr{A}(\omega)\|_{2}$ unless otherwise specified. The reduced eigenvalue optimization problems are solved by means of the support based algorithm in Section 3.1, in particular the Matlab package eigopt (see Section 10 in Mengi et al. (2014)). The largest eigenvalue of the full matrix $\mathscr{A}(\omega)$ at a given $\omega$ is computed by means of eigs in MATLAB.

At least quadratic convergence of the proposed subspace framework in the non-smooth case has been established above. Some of the examples here are meant to confirm the realization of this theoretical result in practice. Depending on double precision gives little insight; we typically observe a quadratic convergence behavior at the last one or two subspace iterations before termination in double precision. To illustrate the order of convergence more accurately and more convincingly, following also the practice in Section 3.3, we perform computations in higher precision by means of the Matlab package Advanpix Multiprecision Computing Toolbox. In the majority of the non-smooth examples below, the computations are performed to ensure 400 decimal digits accuracy, specifically tol $=10^{-400}$ for these examples. We illustrate the quick convergence of the subspace framework in the non-smooth case by plotting $\ln \left(e_{k+1}\right)$ vs. $\ln \left(e_{k}\right)$, as well as $\ln \left(\xi_{k+1}\right)$ vs. $\ln \left(\xi_{k}\right)$ in high precision, where $e_{k}:=\lambda_{*}-\lambda_{\max }\left(\mathscr{A}^{1 / k-1}\left(\omega^{(k)}\right)\right)$ and $\xi_{k}:=\left|\omega^{(k)}-\omega_{*}\right|$ with $\omega_{*}$ denoting the global minimizer of $\lambda_{\max }(\mathscr{A}(\omega))$ and $\lambda_{*}:=\lambda_{\max }\left(\mathscr{A}\left(\omega_{*}\right)\right)$.

Distance to a Nearest Definite Pair. Consider the Hermitian pair $(A, B)$ with

$$
A=\left(\widetilde{G}+\widetilde{G}^{*}\right) / 2 \quad \text { and } \quad B=-\mathrm{i}\left(\widetilde{G}-\widetilde{G}^{*}\right) / 2
$$

and $\widetilde{G}:=G e^{\mathrm{i} \pi / 6}$, where $G$ is the $640 \times 640$ Grcar matrix. An application of the subspace framework for the computation of the inner numerical radius of $A+\mathrm{i} B$ yields the global minimizer $\theta_{*}=$ 2.617992877994 of $\lambda_{\max }(A \cos \theta+B \sin \theta)$, as well as $\lambda_{\max }\left(A \cos \theta_{*}+B \sin \theta_{*}\right)=0.634045490256$. Now as explained at the end of Section 2.2, this implies the pair is not definite and $0 \in F(A+\mathrm{i} B)$; this is confirmed by the plot of $F(A+\mathrm{i} B)$ on the top right in Figure 1 .

We deduce from (2.3) that $d_{\delta}(A, B)=0.644045490256$ for $\delta=10^{-2}$. The right column in Figure 1 illustrates the field of values of $A+\mathrm{i} B,\left(A+\Delta A_{*}\right)+\mathrm{i}\left(B+\Delta B_{*}\right), \widetilde{A}+\mathrm{i} \widetilde{B}=e^{-\mathrm{i} \varphi}\left(\left(A+\Delta A_{*}\right)+\mathrm{i}\left(B+\Delta B_{*}\right)\right)$ for the choice of $\delta=10^{-2}$ and $\varphi=\theta_{*}+\pi / 2$, where $\Delta A_{*}, \Delta B_{*}$ are as in (2.4). Although $\lambda_{\max }\left(A \cos \theta_{*}+\right.$ $B \sin \theta_{*}$ ) is simple, it turns out to be very close to the second largest eigenvalue; these two eigenvalues differ by about $10^{-7}$. The order of convergence of our subspace framework on this example is at least quadratic; this is depicted in Table 4, which lists the iterates of Algorithm 2 with $\varepsilon=10^{-6}$.

Testing Hyperbolicity of Quadratic Eigenvalue Problems. The quadratic eigenvalue problem (QEP)

$$
Q(\lambda) x=\left(\lambda^{2} A+\lambda B+C\right) x=0
$$

is said to be hyperbolic if $A, B, C \in \mathbb{C}^{n \times n}$ are Hermitian, $A$ is positive definite, as well as $\left(x^{*} B x\right)^{2}>$ $4\left(x^{*} A x\right)\left(x^{*} C x\right)$ for all non-zero $x \in \mathbb{C}^{n}$. A hyperbolic QEP possesses appealing properties; for instance a hyperbolic QEP with positive definite $B$ and positive semidefinite $C$ has $n$ linearly independent eigenvectors and $2 n$ real eigenvalues (as discussed in Section 7.6 in Lancaster (1966)). It is well known (see e.g. Higham et al. (2002)) that the hyperbolicity of the QEP in (4.11) is equivalent to the definiteness of $\left(A_{1}, B_{1}\right)$ with

$$
A_{1}=\left[\begin{array}{cc}
-C & 0 \\
0 & A
\end{array}\right], \quad B_{1}=-\left[\begin{array}{cc}
B & A \\
A & 0
\end{array}\right] \text {. }
$$




\begin{tabular}{c|c|c|c}
\hline \hline$k$ & $p$ & $\lambda_{\max }\left(\mathscr{A}^{4 / k}\left(\omega^{(k+1)}\right)\right)$ & $\omega^{(k+1)}$ \\
\hline 4 & 4 & 0.629840138568 & 2.618127739876 \\
5 & 5 & $0 . \underline{63} 2130046510$ & 2.617077493245 \\
6 & 6 & $0 . \underline{634045279755}$ & $2 . \underline{617993771783}$ \\
7 & 8 & $0 . \underline{634045490256}$ & $2 . \underline{617993877986}$ \\
8 & 10 & $0 . \underline{634045490256}$ & $2 . \underline{617993877986}$ \\
\hline \hline
\end{tabular}

Table 4: This table concerns the minimization of $\lambda_{\max }(\mathscr{A}(\omega))$, where $\mathscr{A}(\omega):=A \cos \omega+B \sin \omega$ for Hermitian matrices $A, B$ defined in terms of the $640 \times 640$ Grcar matrix. The quantities $\lambda_{\max }\left(\mathscr{A}^{\mathscr{N} k}\left(\omega^{(k+1)}\right)\right)$ and $\omega^{(k+1)}:=\arg \min _{\omega} \lambda_{\max }\left(\mathscr{A}^{\mathscr{1} k}(\omega)\right)$ by Algorithm 2 starting with $\omega^{(1)}=0.45$ are listed along with $p:=\operatorname{dim} \mathscr{V}_{k}$ with respect to $k$.

Consider in particular the QEP $Q(\lambda)=\lambda^{2} A+\lambda B+C$ that is linked to a damped-mass spring system, where $A, B, C$ are $500 \times 500$ matrices such that $A=I$,

$$
B=\beta\left[\begin{array}{ccccc}
20 & -10 & & & \\
-10 & 30 & \ddots & & \\
& \ddots & \ddots & \ddots & \\
& & \ddots & 30 & -10 \\
& & & -10 & 20
\end{array}\right], \quad C=\left[\begin{array}{cccc}
15 & -5 & & \\
-5 & \ddots & \ddots & \\
& \ddots & \ddots & -5 \\
& & -5 & 15
\end{array}\right]
$$

for a given real number $\beta>0$. We determine the hyperbolicity of the QEP for different $\beta$ values by testing the definiteness of the associated $1000 \times 1000$ pair $\left(A_{1}, B_{1}\right)$ as in (4.12). Table 5 indicates whether the pair $\left(A_{1}, B_{1}\right)$ is definite or not for eight equally spaced $\beta$ values in the interval $[0.500,0.528]$. The number of iterations to compute the inner numerical radius of $A_{1}+\mathrm{i} B_{1}$ (up to the prescribed tolerance tol $=10^{-12}$ ) is eight for each $\beta$ value. This is a non-smooth example; the largest eigenvalue of $A_{1} \cos \theta+B_{1} \sin \theta$ has multiplicity 2 at the minimizing $\theta$ for each $\beta$ value, yet we observe the quadratic convergence of Algorithm 2 consistent with what is expected in theory. This is hinted by Table 6 , which lists the iterates $\lambda_{\max }\left(\mathscr{A}^{\mathscr{T} / k}\left(\omega^{(k+1)}\right)\right)$ and $\omega^{(k+1)}$ with respect to $k$ for $\beta=0.512$ and $\beta=0.524$. In the table the number of accurate digits of $\omega^{(k+1)}$ is not doubled at the last rows for both values of $\beta$. This is because the reduced problems are solved to ensure a prescribed accuracy for the computed value of the minimum $\lambda^{(k+1)}:=\lambda_{\max }\left(\mathscr{A}^{\mathscr{Y} k}\left(\omega^{(k+1)}\right)\right)$, but not for the computed value of the global minimizer $\omega^{(k+1)}$. Even though the values of $\lambda^{(k+1)}$ in the table are at least twelve decimal digits accurate, this is not necessarily true for the values of $\omega^{(k+1)}$. To get a better insight into the order of convergence, we also perform computations with 400 decimal digits accuracy for the pair $\left(A_{1}, B_{1}\right)$ defined as in (4.12), (4.13) with $\beta=0.512$ and $A, B, C$ of size 50 . Here we limit the sizes of $A_{1}, B_{1}$ to 100 , because the computation of the largest eigenvalues using eigs and with the high precision package takes considerable amount of time for larger matrices. Figure 3 indicates that the orders of convergence of $\left\{\lambda_{\max }\left(\mathscr{A}^{1 / k-1}\left(\omega^{(k)}\right)\right)\right\}$ and $\left\{\omega^{(k)}\right\}$ are at least quadratic.

Linear Systems in Saddle Point Form. We consider again the matrix pair $(\mathscr{A}, \mathscr{J})$ discussed in Section 3.3, where $\mathscr{A} \in \mathbb{R}^{(n+m) \times(n+m)}$ is the coefficient matrix of the form (3.9) that originates from a discretization of the Stokes equation, and $\mathscr{J}=\operatorname{diag}\left(I_{n},-I_{m}\right)$. We run our subspace procedure to minimize 


\begin{tabular}{|c|c|c|c|c|}
\hline$\beta$ & 0.500 & 0.504 & 0.508 & 0.512 \\
\hline definite & no & no & no & no \\
\hline
\end{tabular}

\begin{tabular}{|c|c|c|c|c|}
\hline$\beta$ & 0.516 & 0.520 & 0.524 & 0.528 \\
\hline definite & no & yes & yes & yes \\
\hline
\end{tabular}

Table 5: The definiteness of the pair $\left(A_{1}, B_{1}\right)$ defined as in (4.12) associated with the QEP (4.11) for $A, B, C$ as in (4.13) and for several values of $\beta$.

\begin{tabular}{|c|c|c|c|}
\hline \multirow[b]{2}{*}{$k$} & \multirow[b]{2}{*}{$p$} & \multicolumn{2}{|c|}{$\beta=0.512$} \\
\hline & & $\lambda^{(k+1)}$ & $\omega^{(k+1)}$ \\
\hline 4 & 6 & 0.218717671040 & 1.972911641774 \\
\hline 5 & 8 & $0 . \underline{006821928930}$ & $1 . \underline{890604000858}$ \\
\hline 6 & 10 & 0.008594146027 & 1.897161234772 \\
\hline 7 & 12 & 0.008594402114 & 1.897151450236 \\
\hline \multirow[t]{2}{*}{8} & 14 & 0.008594402114 & $1 . \underline{897151450823}$ \\
\hline & & \multicolumn{2}{|c|}{$\beta=0.524$} \\
\hline$k$ & $p$ & $\lambda^{(k+1)}$ & $\omega^{(k+1)}$ \\
\hline 4 & 6 & 0.233188266820 & 1.985354587351 \\
\hline 5 & 8 & $-0 . \underline{006697302959}$ & 1.901962042436 \\
\hline 6 & 10 & -0.004923289259 & 1.908357152861 \\
\hline 7 & 12 & -0.004923056427 & $1 . \overline{908348045018}$ \\
\hline 8 & 14 & $-0 . \overline{004923056427}$ & 1.908348041619 \\
\hline
\end{tabular}

Table 6: The iterates $\lambda^{(k+1)}:=\lambda_{\max }\left(\mathscr{A}^{\mathscr{Y} k}\left(\omega^{(k+1)}\right)\right), \omega^{(k+1)}$, and $p:=\operatorname{dim} \mathscr{V}_{k}$ are listed, when Algorithm 2 is applied to the example concerning the hyperbolicity of a QEP.

$\lambda_{\max }(\mathscr{A} \cos \theta+\mathscr{J} \sin \theta)$ over $\theta \in[0,2 \pi]$. The computed results coincide with the ones obtained from a direct application of Algorithm 1 in Section 3.3. As remarked before, $\lambda_{\max }\left(\mathscr{A} \cos \theta_{*}+\mathscr{J} \sin \theta_{*}\right)$ has multiplicity three at the global minimizer $\theta_{*}$. Our subspace framework again exhibits at least a quadratic convergence; this is evident from Table 7, even more convincing evidence is provided by the plots of the decays of the errors in Figure 4 obtained by requiring 400 decimal digits accuracy in the computations.

Influence of the Parameter $\varepsilon$ on the Order of Convergence. Let us consider $C=\widetilde{C} e^{i \pi / 6}$, where $\widetilde{C}$ is as in (3.8), but now the size of $\widetilde{C}$ is $n=100$. It again turns out that, letting $A:=\left(C+C^{*}\right) / 2$, $B:=-\mathrm{i}\left(C-C^{*}\right) / 2$, the eigenvalue $\lambda_{\max }(\mathscr{A}(\omega):=A \cos \omega+B \sin \omega)$ has multiplicity 2 at the global minimizer $\omega_{*}$. We run Algorithm 2 to minimize $\lambda_{\max }(\mathscr{A}(\omega))$ requiring 600 decimal digits accuracy in the computed results by setting $\varepsilon=0$ and $\varepsilon=10^{-16}\left(\|A\|_{2}+\|B\|_{2}\right)$. Figure 5 indicates that, at least on this example, including also the additional eigenvectors corresponding to eigenvalues close to the largest eigenvalue in the subspaces leads to a faster convergence. We have also tested the choices for $\varepsilon$ larger than $10^{-16}\left(\|A\|_{2}+\|B\|_{2}\right)$, e.g., $\varepsilon=10^{-6}$. They yield results nearly the same as $\varepsilon=10^{-16}\left(\|A\|_{2}+\|B\|_{2}\right)$. 

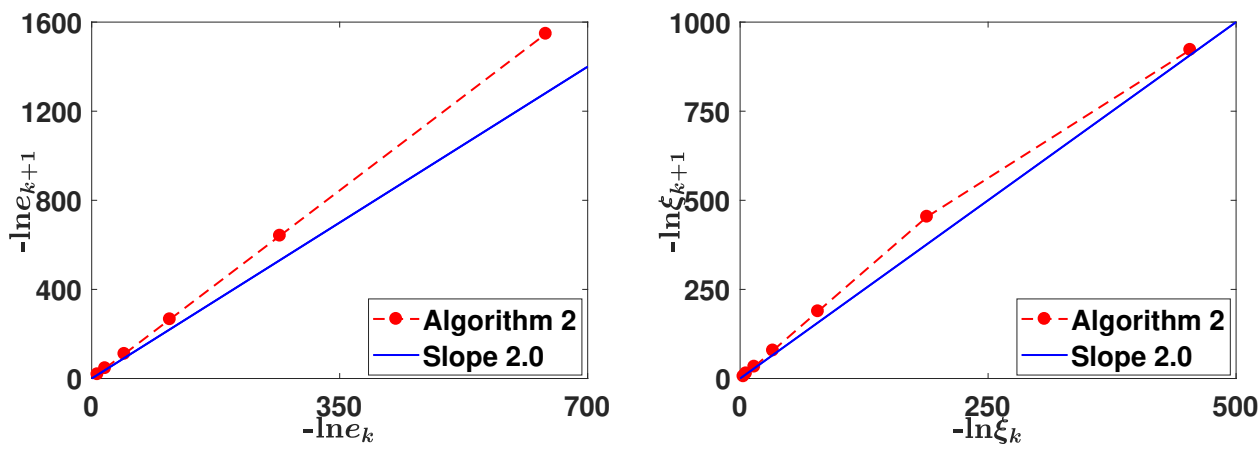

FIG. 3: The orders of convergence of Algorithm 2 for the QEP example with $A, B, C$ defined as in (4.13) of size $n=50$ and for $\beta=0.512$.

\begin{tabular}{c|c|c|c}
\hline \hline$k$ & $p$ & $\lambda_{\max }\left(\mathscr{A}^{1 / k}\left(\omega^{(k+1)}\right)\right)$ & $\omega^{(k+1)}$ \\
\hline 2 & 2 & -0.876669786135 & 2.023688714623 \\
3 & 4 & -0.023285504705 & 3.088556373675 \\
4 & 7 & $-0 . \underline{022225058342}$ & $3 . \underline{087503074213}$ \\
5 & 10 & $-0 . \underline{022224901666}$ & $3 . \underline{087502918535}$ \\
6 & 13 & $-0 . \underline{022224901666}$ & $3 . \underline{087502918535}$ \\
\hline \hline
\end{tabular}

Table 7: This table concerns the positive or negative definiteness of $\mathscr{A}-\mu \mathscr{J}$ for some $\mu$, where $\mathscr{A}$ is the matrix for the saddle point system arising from the Stokes equation, $\mathscr{J}:=\operatorname{diag}\left(I_{n},-I_{m}\right)$. The iterates of Algorithm 2 and $p:=\operatorname{dim} \mathscr{V}_{k}$ are listed when it is applied to minimize $\lambda_{\max }(\mathscr{A} \cos \omega+\mathscr{J} \sin \omega)$.

Performance of the Subspace Framework. We test the performance of Algorithm 2 for the computation of $\zeta\left(A_{n}, B_{n}\right)$, where

$$
A_{n}=\left(C_{n}+C_{n}^{*}\right) / 2, \quad B_{n}=-\mathrm{i}\left(C_{n}-C_{n}^{*}\right) / 2,
$$

$C_{n}=P_{n}+i R_{n}$, the matrix $P_{n}$ denotes the $n \times n$ matrix obtained from the finite difference discretization of the Poisson operator by employing the five-point formula, and $R_{n}$ denotes a random $n \times n$ sparse matrix generated by the Matlab command sprand $(n, n, 20 / n)$. Table 8 lists the computed values of the inner numerical radius by the subspace framework, number of subspace iterations and run-times in seconds to reach the specified accuracy for the pairs $\left(A_{n}, B_{n}\right)$ of sizes varying between 10000 and 90000. The number of subspace iterations, as well as the time to solve the reduced eigenvalue optimization problems, do not vary much with respect to $n$. However, the time required for the computation of the largest eigenvalue of the full problem at every iteration increases with respect to $n$. In essence the total runtime is determined by these large-scale eigenvalue computations for large values of $n$.

\section{Conclusion}

The algorithm in Mengi et al. (2014) based on piece-wise quadratic model functions appears to be quite effective in dealing with global minimization problems involving a non-convex largest eigenvalue 

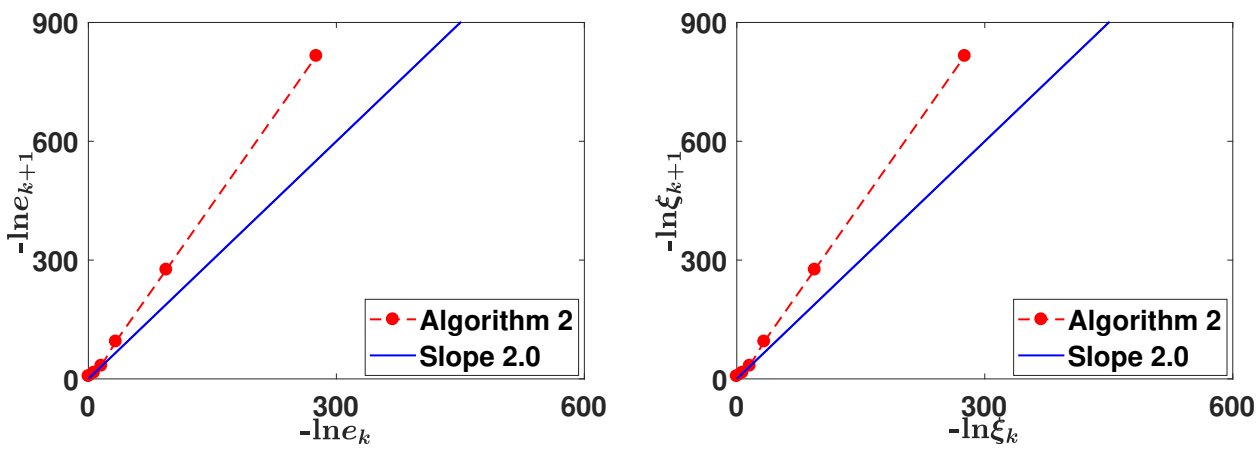

FIG. 4: The order of convergence of Algorithm 2 for the saddle point linear system applied to minimize $\lambda_{\max }(\mathscr{A} \cos \omega+\mathscr{J} \sin \omega)$ where $\mathscr{A}$ is the coefficient matrix for the saddle point system arising from the Stokes equation and $\mathscr{J}:=\operatorname{diag}\left(I_{n},-I_{m}\right)$.
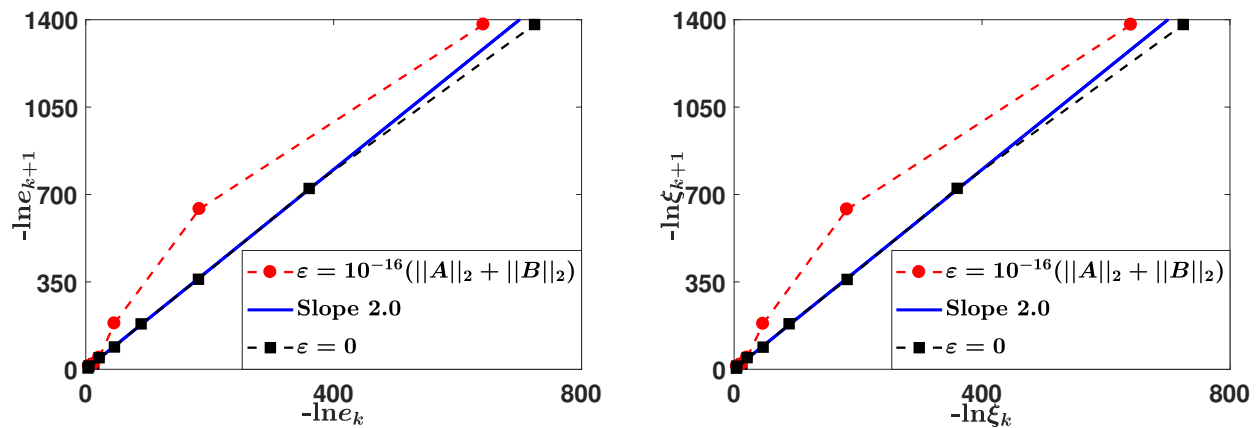

FIG. 5: The effect of $\varepsilon$ on the order of convergence is illustrated. In particular, setting $\varepsilon>0$ (meaning the additional eigenvectors corresponding to nearby eigenvalues close to the largest eigenvalue are also included in the subspaces) leads to a faster convergence compared with $\varepsilon=0$ (meaning only the eigenvector corresponding to the largest eigenvalue is included in the subspace). This example concerns the computation of the inner numerical radius of $C=\widetilde{C} e^{i \pi / 6}$, where $\widetilde{C}$ is as in (3.8) and of size $n=100$.

\begin{tabular}{|c||ccccc|}
\hline$n$ & \# iter & total time & reduced prob & eigval comp & $\zeta\left(A_{n}, B_{n}\right)$ \\
\hline \hline 10000 & 21 & 22.62 & 4.41 & 17.16 & 653.69 \\
22500 & 26 & 110.61 & 6.43 & 100.96 & 983.80 \\
40000 & 24 & 168.44 & 5.91 & 156.91 & 1316.77 \\
62500 & 20 & 315.34 & 13.18 & 291.97 & 1667.44 \\
90000 & 21 & 594.99 & 11.12 & 569.67 & 1995.49 \\
\hline
\end{tabular}

Table 8: The performance of the subspace framework (Algorithm 2) to compute $\zeta\left(A_{n}, B_{n}\right)$ for $A_{n}, B_{n} \in$ $\mathbb{R}^{n \times n}$ as in (4.14) in terms of $C_{n}=P_{n}+\mathrm{i} R_{n}$, the Poisson matrix $P_{n}$ and the sparse random matrix $R_{n}$. The number of subspace iterations, total run-time, times for the reduced problems, large-scale eigenvalue computations in seconds are given in the 2nd, 3rd, 4th, 5th columns, respectively. 
function of a Hermitian matrix depending on one parameter. On the other hand, the subspace framework in Kangal et al. (2018) is quite effective to deal with such problems when the Hermitian matrix is large. It accurately reduces the large dimensionality by projecting the Hermitian matrix to small subspaces formed of eigenvectors. Here we have illustrated the efficiency of these algorithms on the computation of the inner numerical radius.

As a by-product, we have generalized the subspace framework of Kangal et al. (2018) to better cope with non-smoothness at the minimizer. The generalized subspace framework adds not only the eigenvector corresponding to the largest eigenvalue, but also the eigenvectors corresponding to nearby eigenvalues.

We have proven rapid convergence results for both algorithms in the non-smooth case when the largest eigenvalue is not simple at the minimizer. The algorithm in Mengi et al. (2014) is shown to generate a sequence $\left\{\ell^{(k)}\right\}$ of lower bounds such that both $\left\{\ell^{(2 k)}\right\}$ and $\left\{\ell^{(2 k+1)}\right\}$ converge to the globally smallest value of the largest eigenvalue function at a quadratic rate. The generalized subspace framework is shown to generate a sequence of iterates $\left\{\omega^{(k)}\right\}$ that converges to the global minimizer at a quadratic rate. What we witness in practice is consistent with these theoretical findings. To this end, several numerical results concerning the inner numerical radius computation in the non-smooth case are reported confirming the expected in theory.

Acknowledgements. The authors are grateful to two anonymous referees for reading the initial version of this manuscript carefully, and providing constructive feedback.

\section{REFERENCES}

Aliyev, N., Benner, P., Mengi, E., Schwerdtner, P. \& Voigt, M. (2017) Large-scale computation of $\mathscr{L}_{\infty}$-norms by a greedy subspace method. SIAM J. Matrix Anal. Appl., 38, 1496-1516.

BOYD, S. \& BALAKRISHNAN, V. (1990) A regularity result for the singular values of a transfer matrix and a quadratically convergent algorithm for computing its $L_{\infty}$-norm. Syst. Control Lett., 15, 1-7.

Breiman, L. \& Cutler, A. (1993) A deterministic algorithm for global optimization. Math. Program., 58, 179-199.

Cheng, S.-H. \& Higham, N. J. (1999) The nearest definite pair for the Hermitian generalized eigenvalue problem. Linear Algebra Appl., 302-303, 63-76.

Clarke, F. H. (1990) Optimization and Nonsmooth Analysis. Philadelphia, PA, USA: Society for Industrial and Applied Mathematics.

Davies, P. I., Higham, N. J. \& Tisseur, F. (2001) Analysis of the Cholesky method with iterative refinement for solving the symmetric definite generalized eigenproblem. SIAM J. Matrix Anal. Appl., 23, 472-493.

Elman, H. C., Ramage, A. \& Silvester, D. J. (2007) Algorithm 866: IFISS, a MATLAB toolbox for modelling incompressible flow. ACM Trans. Math. Softw., 33, Art. 14.

Guo, C. H., Higham, N. J. \& Tisseur, F. (2009) An improved arc algorithm for detecting definite Hermitian pairs. SIAM J. Matrix Anal. Appl., 31, 1131-1151.

He, C. \& Watson, G. A. (1997) An algorithm for computing the numerical radius. IMA J. Numer. Anal., 17, 329-342.

Helmberg, C., Overton, M. \& Rendl, F. (2014) The spectral bundle method with second-order information. Optim. Method Softw., 29, 855-876.

Helmberg, C. \& Rendl, F. (2000) A spectral bundle method for semidefinite programming. SIAM J. Optim., 10, 673-696.

Higham, N. J., Tisseur, F. \& VAn Dooren, P. (2002) Detecting a definite Hermitian pair and a hyperbolic or elliptic quadratic eigenvalue problem, and associated nearness problems. Linear Algebra Appl., 351, 455-474.

Kangal, F., Meerbergen, K., Mengi, E. \& Michiels, W. (2018) A subspace method for large-scale eigen- 
value optimization. SIAM J. Matrix Anal. Appl., 39, 48-82.

KANGAL, F. \& MENGI, E. (2018) Non-smooth rate-of-convergence analyses of algorithms for eigenvalue optimization. arXiv:1805.04393v1 [math.NA]. Math arXiv Preprint.

Kato, T. (1995) Perturbation Theory for Linear Operators. Berlin Heidelberg: Springer-Verlag.

Kressner, D., Lu, D. \& VAndereycken, B. (2018) Subspace acceleration for the Crawford number and related eigenvalue optimization problems. SIAM J. Matrix Anal. Appl., 39, 961-982.

Kressner, D. \& VAndereycken, B. (2014) Subspace methods for computing the pseudospectral abscissa and the stability radius. SIAM J. Matrix Anal. Appl., 35, 292-313.

LANCASter, P. (1964) On eigenvalues of matrices dependent on a parameter. Numer. Math., 6, 377-387.

LANCASTer, P. (1966) Lambda-matrices and vibrating systems. Pergamon Press, Oxford.

Liesen, J. \& PARLETT, B. N. (2008) On nonsymmetric saddle point matrices that allow conjugate gradient iterations. Numer. Math., 108, 605-624.

Meerbergen, K., Michiels, W., Van Beeumen, R. \& Mengi, E. (2017) Computation of pseudospectral abscissa for large-scale nonlinear eigenvalue problems. IMA J. Numer. Anal., 37, 1831-1863.

Mengi, E., Yildirim, E. A. \& Kilic, M. (2014) Numerical optimization of eigenvalues of Hermitian matrix functions. SIAM J. Matrix Anal. Appl., 35, 699-724.

MENGI, E. (2017) A support function based algorithm for optimization with eigenvalue constraints. SIAM J. Optim., 27, 246-268.

Mengi, E. \& Overton, M. L. (2005) Algorithms for the computation of the pseudospectral radius and the numerical radius of a matrix. IMA J. Numer. Anal., 25, 648-669.

Rellich, F. (1969) Perturbation Theory of Eigenvalue Problems. Gordon and Breach.

Sirkovic, P. \& KRESSNER, D. (2016) Subspace acceleration for large-scale parameter-dependent Hermitian eigenproblems. SIAM J. Matrix Anal. Appl., 37, 695-718.

StewART, G. W. (1979) Pertubation bounds for the definite generalized eigenvalue problem. Linear Algebra Appl., 23, 69-85. 